\title{
MT5-MMP controls APP and $\beta$-CTF/C99 metabolism through proteolytic-dependent and -independent mechanisms relevant for Alzheimer's disease
}

\author{
Laura García-González $^{1} \quad$ Jean-Michel Paumier ${ }^{1} \quad$ Laurence Louis ${ }^{1} \quad$ Dominika Pilat \\ Anne Bernard $^{1}$ ｜ Delphine Stephan ${ }^{1}$ | Nicolas Jullien ${ }^{1}$ | Frédéric Checler ${ }^{2}$ | \\ Emmanuel Nivet $^{1} \quad$ Michel Khrestchatisky ${ }^{1} \mid$ Kévin Baranger $^{1} \quad$ Santiago Rivera $^{1}$ (D) \\ ${ }^{1}$ Aix-Marseille Univ, CNRS, INP, Inst Neurophysiopathol, Marseille, France \\ ${ }^{2}$ IPMC, UMR 7275 CNRS-UCA, Labex DistAlz, Valbonne, France
}

\author{
Correspondence \\ Kévin Baranger and Santiago Rivera, UMR \\ 7051, CNRS - Aix-Marseille Université, \\ Institute of Neuropathophysiology (INP), \\ Campus Timone, 27 Bld Jean Moulin, \\ 13005 Marseille, France. \\ Email: kevin.baranger@univ-amu.fr (K. B.) \\ and santiago.rivera@univ-amu.fr (S. R.)

\section{Present address \\ Jean-Michel Paumier, Department of Neurology, Northwestern University Feinberg School of Medicine, Chicago, IL, USA \\ Funding information \\ CNRS; Aix-Marseille Université; French National Research Agency (ANR); CoEN; Fondation Plan Alzheimer; France Alzheimer; Vaincre l'Alzheimer; Fonds Européens de Développement Régional; La fondation NRJ-Institut de France; Fondation pour la Recherche Médicale, Grant/Award Number: FDT201904008423 and FDT202001011017;}

\begin{abstract}
We previously discovered the implication of membrane-type 5-matrix metalloproteinase (MT5-MMP) in Alzheimer's disease (AD) pathogenesis. Here, we shed new light on pathogenic mechanisms by which MT5-MMP controls the processing of amyloid precursor protein (APP) and the fate of amyloid beta peptide (A $\beta$ ) as well as its precursor C99, and C83. We found in human embryonic kidney cells (HEK) carrying the APP Swedish familial mutation (HEKswe) that deleting the C-terminal non-catalytic domains of MT5-MMP hampered its ability to process APP and release the soluble $95 \mathrm{kDa}$ form (sAPP95). Catalytically inactive MT5-MMP variants increased the levels of $\mathrm{A} \beta$ and promoted APP/C99 sorting in the endolysosomal system, likely through interactions of the proteinase C-terminal portion with C99. Most interestingly, the deletion of the C-terminal domain of MT5-MMP caused a strong degradation of $\mathrm{C} 99$ by the proteasome and prevented $\mathrm{A} \beta$ accumulation. These discoveries reveal new control of MT5-MMP over APP by proteolytic and non-proteolytic mechanisms driven by the C-terminal domains of the proteinase. The targeting of these non-catalytic domains of MT5-MMP could, therefore, provide new insights into the therapeutic regulation of APP-related pathology in AD.
\end{abstract}

\footnotetext{
Abbreviations: 3xTG, transgenic mice expressing human APP, MAPT, and PSEN1 genes with three familial mutations; 5xFAD, transgenic mice expressing human APP and PSEN1 genes with five familial mutations; AD, Alzheimer's disease; ADAM, a disintegrin and metalloprotease; APP, amyloid protein precursor; A $\beta$, beta-amyloid peptide; BACE-1, beta-site APP-cleaving enzyme-1; C3, BACE-1 inhibitor IV; C99/C83, APP CTF of 99/83 amino acids; $\mathrm{CAT}^{-}$, deletion of the catalytic domain; CTF, C-terminal fragment; DAPT, $\gamma$-secretase inhibitor; EXT'- deletion of the extracellular domain; GFP, green fluorescent protein; HEKswe, human embryonic kidney cells carrying the APP Swedish mutation; HPX; deletion of the hemopexin domain; HRP, horseradish peroxidase enzyme; IC', deletion of the intracytoplasmic domain; MMP, matrix metalloproteinase; MT1/5-MMP, membrane-type 1/5 matrix metalloproteinase; MT1/5 -MMP, catalytically inactive MT1-MMP or MT5-MMP; MTT, 3-(4,5-Dimethylthiazol-2yl)-2,5-diphenyl tetrazolium bromide; PSEN 1(2), presenilin 1 and 2; qPCR, quantitative polymerase chain reaction; RFP, red fluorescent protein; RXP03, MMP inhibitor; sAPP95, soluble amyloid protein precursor fragment generated by MT5-MMP; sAPPFL, full-length soluble amyloid protein precursor; sAPP $\alpha / \beta$, soluble APP $\alpha / \beta$; Tf, transferrin; TIMP, tissue inhibitor of MMPs; TIMP-1 $\Delta$, inactive TIMP-1; TM/IC ${ }^{-}$, deletion of the transmembrane and intracytoplasmic domains.
} 
Conseil Général des Alpes Maritimes; LABEX DISTALZ; A*Midex foundation, Grant/Award Number: ANR-17-EURE-0029; French National Research Agency

\section{K E Y W O R D S}

amyloid beta precursor protein, endosome, metalloproteinase, proteasome, secretase

\section{INTRODUCTION}

Deciphering relevant pathophysiological mechanisms of $\mathrm{AD}$ on which to base an effective therapy represents a major scientific, societal, and economic challenge. A $\beta$ resulting from the cleavage of APP, has long been considered to be the major pathogenic factor in $\mathrm{AD}$, but other neurotoxic APP fragments are emerging in a proteolytic context more diverse than expected (reviewed in 1,2). APP cleavage by beta-site APP-cleaving enzyme 1 (BACE1/ $\beta$-secretase) generates a transmembrane $\mathrm{C}$-terminal fragment of 99 amino acids ( $\beta$-CTF/C99), then cleaved by $\gamma$-secretase to release $\mathrm{A} \beta$ monomers. Their assembly and accumulation into oligomers and fibrils are the cornerstone underlying the pathogenic hypothesis of the amyloid cascade of $\mathrm{AD} .^{3}$ Although much less studied, the toxicity of the immediate precursor of $\mathrm{A} \beta, \mathrm{C} 99$, has also been documented. ${ }^{4,5} \mathrm{C} 99$ accumulates early in the brains of $\mathrm{AD} 3 \mathrm{xTg}$ and $5 \mathrm{xFAD}$ mouse models, even before A $\beta .^{6,7} \mathrm{C} 99$ also accumulates in fibroblasts and brains of AD patients, ${ }^{8,9}$ as well as in human neurons derived from induced pluripotent stem cell (iPS) harboring $\mathrm{AD}$ familial mutations, which show endosomal dysfunction and altered subcellular trafficking. ${ }^{10}$ Taken together, these data highlight the involvement of C99 in the pathophysiology of AD and justify the study of new mechanisms that could shed light on the modulation of its cellular fate.

Membrane-type 5-matrix metalloproteinase (MT5MMP, also known as MMP-24 or $\eta$-secretase), is one of the newly discovered APP-cleaving enzymes. ${ }^{11}$ Primarily expressed in neural cells, ${ }^{12,13}$ MT5-MMP contributes to physiological and pathological processes in the nervous system. ${ }^{14,15}$ The presence of MT5-MMP in amyloid plaques of $\mathrm{AD}$ patients suggested its possible implication in the disease. ${ }^{13}$ This was further supported by the finding that MT5-MMP deletion in the 5xFAD mouse model of $\mathrm{AD}$ caused drastic reductions in the levels of $\mathrm{C} 99, \mathrm{~A} \beta$, and neuroinflammatory mediators, while prevented the deficits in long-term potentiation (LTP) and cognition, without changes in the activities of $\beta$ - or $\gamma$-secretase. ${ }^{16,17}$ In the same studies, we showed that MT5-MMP promoted the release of a soluble $\mathrm{N}$-terminal fragment of $\sim 95 \mathrm{kDa}$, named sAPP95, and the increase of $A \beta$ and $C 99$ levels in HEKswe cells. ${ }^{16,17}$ Importantly, the reduction of sAPP95 levels in the brains of MT5-MMP-deficient 5xFAD mice, indicated that APP is a genuine in vivo substrate for the proteinase. ${ }^{16,17}$ Moreover, it was reported that MT5-MMP cleavage of APP at the $\eta$-site, combined with cleavage at the $\alpha$-site by ADAM10 released a synaptotoxic APP fragment (ie, A $\eta-\alpha) .{ }^{18}$ In conclusion, MT5-MMP contributes to functional alterations by promoting the formation/accumulation of various toxic APP metabolites, and therefore emerges as a potential new target in $\mathrm{AD} .{ }^{19}$

In this study, we sought to better understand the mechanisms by which MT5-MMP influences the metabolism of APP. Using molecular and cell biology as well as structurefunction approaches, we reveal new proteolytic and nonproteolytic actions of specific domains of MT5-MMP that control the levels of sAPP95, C99, and A $\beta$. This work provides new insights on the molecular mechanisms that support the pathogenic actions of MT5-MMP in AD and broadens the range of possible therapeutic strategies to target this proteinase.

\section{2 | MATERIALS AND METHODS}

\section{1 | Reagents}

We used MMP-2 inhibitor III and BACE inhibitor IV (C3) (both from Millipore, Molsheim, France) and the $\gamma$-secretase inhibitor DAPT (Tocris, Bio-Techne, Lille, France) at $10 \mu \mathrm{M}$, proteasome inhibitor MG132 (Enzo Life Science, Lyon, France) at $5 \mu \mathrm{M}$, bafilomycin A1 (Sigma-Aldrich, Saint-Quentin Fallavier, France) at $50 \mathrm{nM}$, and broad spectrum metalloproteinase inhibitor RxP03 that spares adamalysins $^{20,21}$ at $10 \mu \mathrm{M}$. All the restriction enzymes were purchased from New England Biolabs (Evry, France). All the chemical reagents were from analytic grade and purchased from Sigma-Aldrich, and all the products/media required for cell culture were from Thermo Fisher Scientific (Villebon-sur-Yvette, France), unless otherwise stated.

\section{2 | Plasmid constructs}

cDNAs encoding MMP24 (MT5-MMP), MMP14 (MT1MMP), TIMP1 (TIMP-1), and TIMP2 (TIMP-2) were amplified by PCR from P15 C57B16 mice cerebellum mRNA. MT5-MMP (MT5/GFP), active and inactive MT1MMP (MT1/GFP and MT1 $\Delta /$ GFP), and TIMP-1/RFP and TIMP-2/RFP were cloned and expressed as previously reported. $^{22,23}$ To generate inactive MT5-MMP (MT5 $\Delta /$ GFP), the Quick Change Lighting MultiSite-directed Mutagenesis 
kit (Agilent Technologies, Les Ulis, France) was used and the primers were as follows: MT5 $($ E256Q)For CTG CTG GCC GTG CAT CAA CTG GGC CAT GCA CTG and MT5 $(E 256 Q) R e v$ CAG TGC ATG GCC CAG TTG ATG CAC GGC CAC CAG. All the genes were cloned in pEGFP-N1 or pDSRed II-N1.$^{22,23}$ Plasmids within a pcDNA3.1 backbone encoding full-length active (MT5FL) and inactive (MT5 $\Delta$ ) MT5-MMP and the truncated MT5MMP constructs $\left(\mathrm{CAT}^{-}, \mathrm{HPX}^{-}, \mathrm{EXT}^{-}, \mathrm{TM}^{-} \mathrm{IC}^{-}\right.$, and $\mathrm{IC}^{-}$ ) were synthesized and produced by GeneArt (Thermo Fisher Scientific). All these constructs were fused with a Flag tag on their N-terminal part (Figure 4A). Full-length active MT1-MMP (MT1FL), inactive (MT1 $\Delta$ ), and the truncated construct $\left(\mathrm{IC}^{-}\right)$were cloned in pcDNA3.1 vector and kindly provided by Pr Motoharu Seiki and Pr Takeharu Sakamoto. ${ }^{24}$ The plasmid that encodes human C99 (pcDNA3.1-C99) was previously described. ${ }^{25}$ We used the pcDNA3.1-C99 construct as a template to generate a pcDNA3.1-C99 with HA tag on the N-terminal (HA-C99), using the SLIC method. ${ }^{26}$ All the plasmids were amplified in $E$ coli DH5 $\alpha$ (Thermo Fisher Scientific) and purified using the NucleoBond Xtra Midi Plus EF (MachereyNagel, Hoerdt, France), according to the manufacturer's recommendations.

\section{3 | Transfection of HEK cells}

GFP, MT5/GFP, and MT5 $\Delta /$ GFP encoding plasmids were transiently transfected in HEK cells stably expressing human APP with the Swedish mutation (HEKswe) under the control of the CMV promoter, as previously described. ${ }^{16,17,21}$ Cells were plated to $10^{6}$ cells/well for 24 hours in 6-well plate in DMEM Glutamax, 10\% FBS, $1 \%$ penicillin/streptomycin (P/S), and transfected with $1 \mu \mathrm{g}$ of plasmids, using the Jet Pei transfection reagent (Ozyme, Saint-Quentin En Yvelines, France). Sixteen hours after transfection, the medium was replaced with OptiMEM (serum-free medium) containing $1 \%$ insulin-transferrinselenium (ITS) and cells were then allowed to secrete for 48 hours.

Plasmids ( $0.5 \mu \mathrm{g} / \mathrm{plasmid})$ encoding MT5FL, MT5 $\Delta$, and the different MT5-MMP truncated variants $\mathrm{CAT}^{-}, \mathrm{HPX}^{-}$, $\mathrm{EXT}^{-}, \mathrm{TM} / \mathrm{IC}^{-}$, and $\mathrm{IC}^{-}$or those derived from MT1-MMP that is, MT1FL, MT1 $\Delta$, and $\mathrm{IC}^{-}$were co-transfected with C99 or HA-C99 $(0.5 \mu \mathrm{g} /$ plasmid $)$ in HEK cells using the Jet Pei transfection reagent. Cells were plated to $10^{6}$ cells/well for 24 hours in 6-well plate in DMEM Glutamax, 10\% FBS, 1\% $\mathrm{P} / \mathrm{S}$ and 16 hours after transfection, the medium was replaced with OptiMEM (serum-free medium) containing 1\% ITS. Cells were then treated for 48 hours in the presence or not of DAPT, MG132, and bafilomycin A1 or DMSO (0.01\%), used as control vehicle.

\section{4 | Reverse transcription-quantitative polymerase chain reaction (RT-qPCR)}

Total RNA was extracted from HEKswe cells using the Nucleospin RNA kit (Macherey-Nagel), according to the manufacturer's recommendations. Single-stranded cDNA was synthesized from $1 \mu \mathrm{g}$ of RNA using High-Capacity RNA to cDNA kit (Thermo Fisher Scientific) suitable for quantitative PCR (qPCR). Twenty-five nanograms of cDNA were submitted to qPCR reaction using the Fast Real-Time PCR System (Thermo Fisher Scientific). For each experiment, cDNA samples were analyzed in duplicate and relative gene expression was obtained using the comparative $2^{-(\Delta \Delta \mathrm{Ct})}$ method after normalization to the Gapdh housekeeping gene. ${ }^{7,21}$ All the probes were purchased from Thermo Fisher Scientific (Table 1).

\section{5 | Cell viability}

Cell viability was evaluated using the 3-(4,5-dimethylthi azol-2yl)-2,5-diphenyl tetrazolium bromide (MTT) assay

TABLE 1 Human TaqMan probes used for quantitative PCR analysis

\begin{tabular}{|c|c|c|}
\hline Gene name & Gene description & Probes ID \\
\hline$M M P 2$ & Matrix metalloproteinase 2 & Hs01548727_m1 \\
\hline$M M P 9$ & Matrix metalloproteinase 9 & Hs00234579_m1 \\
\hline$M M P 24$ & $\begin{array}{l}\text { Membrane-type } 5 \text { matrix } \\
\text { metalloproteinase }\end{array}$ & Hs01084645_m1 \\
\hline MMP14 & $\begin{array}{l}\text { Membrane-type } 1 \text { matrix } \\
\text { metalloproteinase }\end{array}$ & Hs01037009_g1 \\
\hline TIMP1 & $\begin{array}{l}\text { Tissue inhibitor of matrix } \\
\text { metalloproteinase } 1\end{array}$ & Hs00171558_m1 \\
\hline$B A C E 1$ & $\begin{array}{l}\text { Beta-site APP-cleaving } \\
\text { enzyme-1 }\end{array}$ & Hs01121195_m1 \\
\hline PSEN1 & Presenilin 1 & Hs00997789_m1 \\
\hline PSEN2 & Presenilin 2 & Hs01577197_m1 \\
\hline PSENEN & $\begin{array}{l}\text { Presenilin enhancer } 2 \\
\text { homolog }\end{array}$ & Hs00708570_s1 \\
\hline APHIA & $\begin{array}{l}\text { Aph1a gamma secretase } \\
\text { subunit }\end{array}$ & Hs01046142_g1 \\
\hline NCSTN & Nicastrin & Hs00950933_m1 \\
\hline$M M E$ & $\begin{array}{l}\text { Membrane metallo- } \\
\text { endopeptidase (neprilysin) }\end{array}$ & Hs00153510_m1 \\
\hline$C T S D$ & Cathepsin D & Hs00157205_m1 \\
\hline$A P P$ & $\begin{array}{l}\text { Amyloid beta (A4) } \\
\text { precursor protein }\end{array}$ & Hs00169098_m1 \\
\hline$G A P D H$ & $\begin{array}{l}\text { Glyceraldehyde } \\
\text { 3-phosphate } \\
\text { dehydrogenase }\end{array}$ & Hs02758991_g1 \\
\hline
\end{tabular}


(Sigma-Aldrich), which measures the mitochondrial activity in living cells. Data were calculated as the percentage of living cells $=\left(\right.$ transfected cell $\mathrm{OD}_{550} /$ control cell $\left.\mathrm{OD}_{550}\right) \times 100$.

\section{6 | Western blot and immunoprecipitation assay}

Supernatants from HEKswe or HEK cells were collected 48 hours after transfection with the different MT5-, MT1MMP and C99 constructs, and centrifuged at $300 \mathrm{~g}$ for 5 minutes at $4^{\circ} \mathrm{C}$. Cells were scraped in RIPA buffer (SigmaAldrich) containing proteinase inhibitors (Millipore) and sonicated. Protein concentration was determined using a Bio-Rad $D C$ protein assay kit (Bio-Rad, Marnes-LaCoquette, France). Proteins $(30 \mu \mathrm{g})$ were loaded on 10\%-15\% SDS-PAGE gels, low molecular weight Tris-Tricine gels or pre-casted 4\%-20\% gels (Thermo Fisher Scientific), and transferred onto nitrocellulose membranes (GE Healthcare, Dutscher, Brumath France). After blocking, membranes were probed with the following primary antibodies: APP $22 \mathrm{C} 11$ (1/1000, Millipore), APP-CTF (1/1000, Sigma-Aldrich), APP 6E10 (1/1000, Ozyme), anti-HA (1/1000, Roche Diagnostics, Meylan, France), anti-FlagM2 (1/1000, SigmaAldrich), anti-MT1-MMP (Abcam, Cambridge, UK), antiGFP (1/2000, Roche Diagnostics), and anti-actin (1/5000, Sigma-Aldrich). Then, the appropriate HRP-conjugated secondary IgG antibodies were used (Jackson Immunoresearch, Interchim, Montluçon, France). Actin was used as loading and normalizing control for $10 \%$ acrylamide gels, while ponceau $\mathrm{S}^{27,28}$ was used as loading and normalizing control for $15 \%$ acrylamide gels.

For the immunoprecipitation (IP) assay, $250 \mu \mathrm{g}$ of protein (cell lysates) in $500 \mu \mathrm{L}$ of RIPA buffer was incubated overnight at $4{ }^{\circ} \mathrm{C}$ with unspecific mouse/rabbit/rat IgG antibodies (Jackson Immunoresearch) or rat anti-HA-tag (Roche Diagnostics) or mouse anti-FlagM2 and rabbit anti-APP-CTF (Sigma-Aldrich) at $4 \mathrm{ng} / \mu \mathrm{L}$ and then pulled-down for 2 hours using $50 \mu \mathrm{L}$ of protein G-coupled dynabeads (Thermo Fisher Scientific). After washing three times with $0.3 \mathrm{M} \mathrm{NaCl}$ solution, samples were denatured and subjected to western blot (WB) using APP-CTF, anti-FlagM2, or anti-HA antibodies, and the corresponding horseradish peroxidase-conjugated secondary IgG antibodies (Jackson Immunoresearch). The EasyBlocker kit was used to limit unspecific signal according to the manufacturer's recommendations (GeneTex, Euromedex, Souffelweyersheim, France).

In all cases, immunodetection was performed using the ECL kit according to the manufacturer's instructions (GE Healthcare, Dutscher, Brumath, France), with an Alliance 9.7-89 EPI apparatus (UVITEC Cambridge, Dutscher). Optical densities were measured using the ImageJ/Fiji software (NIH) and all the optical density plots represent normalized values to loading controls, as indicated in the figure legends.

\subsection{In vitro APP cleavage}

To test whether sAPP95 may result from proteolysis of full-length sAPP (sAPPFL) by MT5-MMP, $2 \mathrm{nM}$ of recombinant catalytic domain of MT5-MMP (cdMT5, Enzo Life Science) was incubated with supernatants from GFPtransfected HEKswe naturally enriched in SAPPFL at $37^{\circ} \mathrm{C}$ in activity buffer $(50 \mathrm{mM}$ Tris- $\mathrm{HCl} \mathrm{pH} 7.5,150 \mathrm{mM} \mathrm{NaCl}$, $5 \mathrm{mM} \mathrm{CaCl}_{2}$, and $0.025 \%$ Brij35) for 5 minutes, 1 hour and 18 hours, as previously described. ${ }^{21}$ Samples were loaded on $10 \%$ SDS-PAGE gels and incubated with $22 \mathrm{C} 11$ antibody as described above.

\section{8 | Immunocytochemistry and pulse labeling}

To perform immunocytochemistry, $6.10^{4}$ HEK cells/well were plated on coverslips for 24 hours and then transfected for 48 hours with control pcDNA3.1 or plasmids encoding the MT5-MMP variants described above. Endosomes were pulse labeled for 30 minutes with $0.25 \mathrm{mg} / \mathrm{mL}$ Alexa Fluor $^{647}$-transferrin (Thermo Fisher Scientific). Cells were then fixed for 15 minutes with Antigenfix solution (Diapath, MM France, Brignais, France) at room temperature. Immunocytochemistry for all antibodies was performed as follows: after washing in PBS 1X, cells were blocked for 1 hour at room temperature using a blocking solution of PBS 1X, 0.1\% Triton X-100, and 3\% BSA. Cells were then incubated in the blocking solution overnight at $4^{\circ} \mathrm{C}$ with the primary antibodies APP 6E10 and anti-A $\beta 40$ (Ozyme), anti-GFP (Roche Diagnostics), anti-EEA1 (Abcam), and anti-FlagM2 (Sigma-Aldrich), followed by appropriate Alexa Fluor-conjugated secondary antibodies for 1 hour at room temperature (Thermo Fisher Scientific). For LAMP-1 (Abcam) immunostaining, we used PBS $1 \mathrm{X}$ containing $0.1 \%$ saponin and 3\% BSA. Nuclei were stained with Hoechst $(0.5 \mu \mathrm{g} / \mathrm{mL}$, Thermo Fisher Scientific). Omission of the primary antibody was used as control and no immunostaining was observed. Coverslips were mounted using Prolong Gold Antifading reagent on Superfrost glass slides (Thermo Fisher Scientific). Images were taken and processed using a confocal microscope (LSM 700) and Zen software (Zeiss, Jena, Germany). A minimum of 20 cells per experimental group for each of the four independent cultures were analyzed using the JACoP plugin of ImageJ software $(\mathrm{NIH}){ }^{29}$ The percentage over the fluorescence signal of one channel that overlaps with the signal of the other channel was calculated on single confocal planes using the Mander's coefficient. Values were 
normalized to values of the control GFP or pcDNA groups, depending on the experimental conditions.

\section{9 | Gel zymography}

We used gelatin zymography on HEKswe cell supernatants to assess for changes in the levels of MMP-2 and MMP-9, also known as gelatinase A and B, respectively. Equal amounts of serum-free supernatants in non-denaturing and non-reducing conditions were subjected to zymogel according to the manufacturer's recommendations (Thermo Fisher Scientific). Gels were digitized using GeneTools software.

\subsection{0 | ELISA assay for A $\beta$}

Levels of $\mathrm{A} \beta 40$ in supernatants of HEK or HEKswe cells transfected with different MT5-MMP variants and/or C99 constructs or treated with C3 or DAPT were evaluated by ELISA (\#KHB3481, Thermo Fisher Scientific) according to the manufacturer's recommendations.

\subsection{1 | Statistics}

All values represent the means \pm SEM of the number of independent cultures indicated in the figure legends. ANOVA followed by a Fisher's LSD post hoc test was used to compare more than two groups. The Student's $t$ test was used to compare two groups. Statistical significance was set up to $P<$.05. Analyses were performed with the GraphPad Prism software (San Diego, California USA, www.graphpad.com).

\section{3 | RESULTS}

\section{1 | MT5-MMP generates the SAPP95 fragment independently of other proteolytic activities}

To investigate the mode of action of MT5-MMP on APP processing, we first asked how it would compare to its close homolog, MT1-MMP, which works in concert with endogenous MMP-2 to generate a soluble APP N-terminal fragment of $95 \mathrm{kDa}$ (sAPP95) in HEKswe cells. ${ }^{21}$ It is noteworthy that the pro-MMP-2 zymogen can be converted to the lower molecular weight active form of MMP-2 upon proteolytic removal of its pro-domain by MT1- and MT5-MMP. ${ }^{30,31}$ As illustrated by gelatin zymography, MT5-MMP (MT5) catalyzed the activation of pro-MMP-2 less efficiently than MT1MMP (Figure 1A). As expected, the inactive MT5-MMP mutant (MT5 $\Delta$ ) failed to activate pro-MMP-2 (Figure 1A).
Before asking whether MT5-MMP could also require MMP-2 activity to process APP, we first confirmed that only MT5 and not MT5 $\Delta$ could release sAPP95 (Figure 1B top panel and Figure 1C). In addition, MT5 did not change the levels of soluble full-length APP (sAPPFL) detected with the 22C11 antibody that recognizes the N-terminal domain of APP (Figure 1B, top panel). On the contrary, MT5 but not MT5 $\Delta$ significantly decreased the content of sAPP $\alpha$ like, as shown by the $6 \mathrm{E} 10$ antibody, which recognizes the $\mathrm{N}$-terminal sequence of human $\mathrm{A} \beta$ and thus, the C-terminal moiety of sAPP $\alpha$-like fragments (Figure 1B middle panel and Figure 1C). This is consistent with the idea that by cleaving APP above the $\alpha$-site, MT5-MMP may compete with $\alpha$-secretase to process APP, resulting in reduced levels of sAPP $\alpha$-like $6 \mathrm{E} 10^{+}$fragments. We evaluated next the functional interactions of MT5-MMP with MMP-2 and found that a chemical MMP-2 inhibitor did not prevent sAPP95 generation by MT5-MMP (Figure 1D). A similar result was obtained with TIMP-1, a potent inhibitor of MMP-2 that spares MT5-MMP (Figure 1E). On the contrary, TIMP-2, which efficiently inhibits both MMP-2 and MT5-MMP, ${ }^{32}$ completely blocked sAPP95 release (Figure 1F). Together, and contrary to MT1-MMP, these data demonstrate no implication of MMP-2 in the processing of APP by MT5MMP. To test the influence of BACE1 (Figure 1G) we used its well-known inhibitor $\mathrm{C} 3$. In addition, $\mathrm{RxP0} 3$ was used as a selective MMP inhibitor that spares ADAMs, that is, $\alpha$ secretase. $^{20,33}$ Only RxP03 significantly inhibited the release of sAPP95 (Figure 1H), thus confirming that MT5-MMP does not require $\beta$-secretase contribution to cleave APP. As the MT5-MMP ectodomain containing the catalytic domain can be physiologically shed from the membrane, ${ }^{34}$ we tested if the soluble recombinant catalytic domain of MT5MMP (cdMT5) could process sAPPFL into sAPP95. After incubating HEKswe cell supernatants enriched in SAPPFL with the cdMT5 for 5 or 60 minutes, we barely detected any sAPP95, as opposed to the control condition with MT5MMP-expressing cells. Instead, the cdMT5 completely degraded sAPPFL after 18 hours (1080 minutes) of incubation (Figure 1I), suggesting the need for a cellular environment to finely tune MT5-MMP processing of APP.

\section{2 | MT5-MMP pro-amyloidogenic features involve proteolytic- and non-proteolytic- dependent pathways}

After confirmation of our previous work, ${ }^{16}$ showing that MT5-MMP stimulated A $\beta$ accumulation (Figure 2A,B), we also observed the implication of $\beta$ - and $\gamma$-secretases in the amyloidogenic process as their respective inhibitors, $\mathrm{C} 3$ and DAPT, blocked the pro-amyloidogenic effect of MT5-MMP (Figure 2A,B). 
Considering that some MMPs regulate transcriptional activity, ${ }^{35}$ we tested next whether MT5-MMP could control the expression of genes encoding proteins involved in APP metabolism (Table 2). We studied MMP24 and MMP14, encoding MT5-MMP and MT1-MMP, respectively, APP, $B A C E 1$ as well as genes encoding the members of the $\gamma$ secretase complex presenilin 1 and 2 (PSEN1 and PSEN2), nicastrin $(N C S T N)$, presenilin enhancer 2 homolog

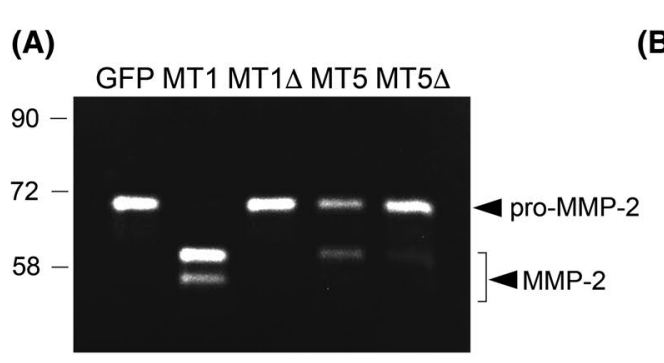

(B)
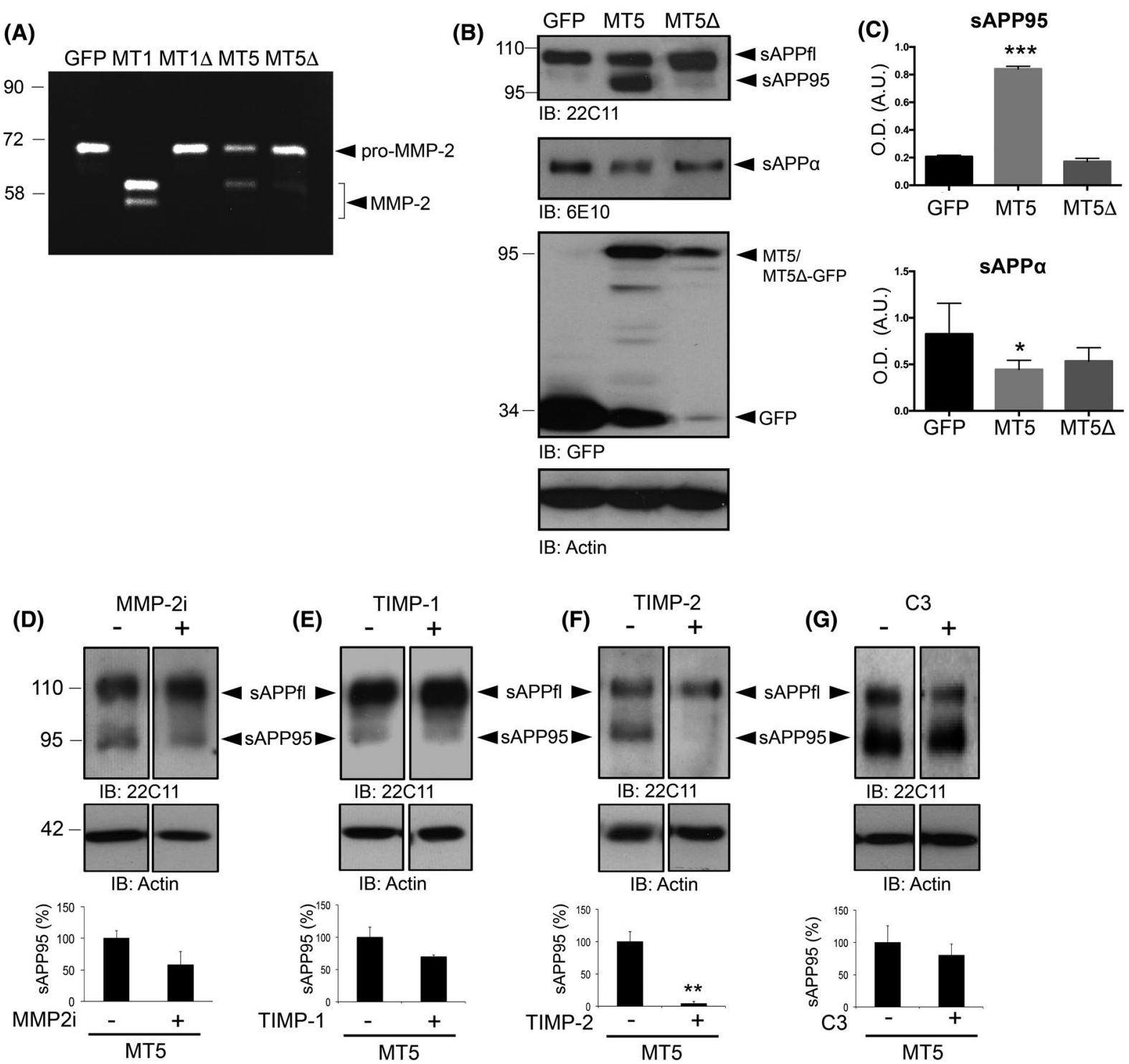

IB: Actin

(H)

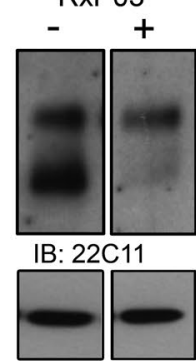

IB: Actin

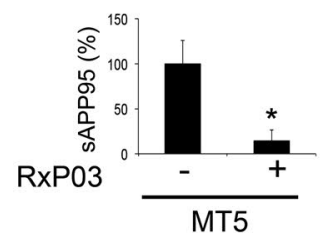

(I)
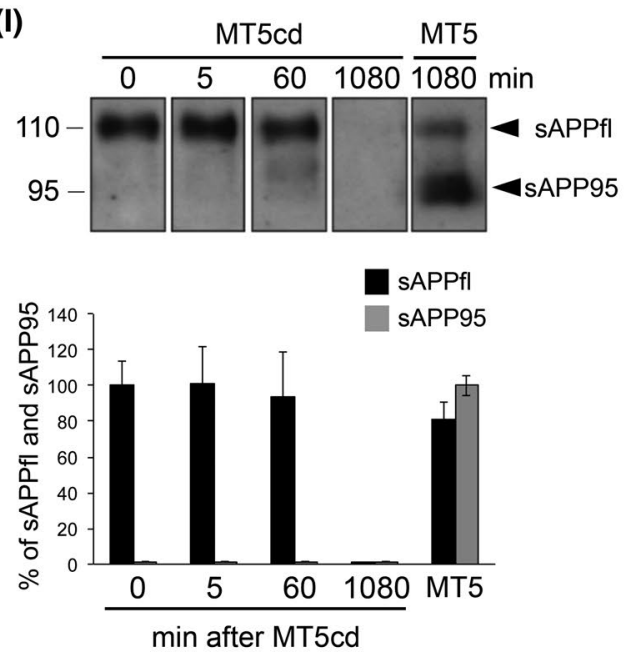
F I G U R E 1 MT5-MMP-mediated generation of sAPP95 is dependent of its catalytic activity. A, Gelatin zymography on supernatants of HEKswe cells 48 hours after transfection with GFP control or active (MT1) or inactive (MT1 $\Delta$ ) MT1-MMP/GFP, and active (MT5) or inactive (MT5 $\Delta$ ) MT5-MMP/GFP constructs. Note that MT5 is less effective than MT1 in activating MMP-2. B, Representative western blots (WB) of sAPPFL and sAPP95 immunoreactivity in HEKswe supernatants using anti-APP 22C11 (top), and WB of sAPP $\alpha$ (second from top) using anti-A $\beta 6 \mathrm{E} 10$ antibodies. The third panel from the top represents WB of GFP and active and inactive MT5-MMP/GFP expression in cell lysates 48 hours after transfection using anti-GFP antibody. The bottom panel represents actin-loading controls. C, Histograms represent the actin-normalized quantifications for sAPP95 (top) and sAPP $\alpha$ (bottom). D-H, WB and the corresponding actin-normalized quantifications of SAPPFL and sAPP95 levels using the anti-APP 22C11 antibody in the supernatants of HEKswe cells after transfection with active MT5-MMP/ GFP (MT5) in the presence or not of $10 \mu \mathrm{M}$ of MMP-2 selective inhibitor III (MMP-2i) (D), TIMP-1/RFP (co-transfection) (E), TIMP-2/RFP (co-transfection) (F), $10 \mu \mathrm{M}$ of C3 (G), and RXP03 (H). I, WB and the corresponding quantifications of immunoreactive bands for the anti-APP $22 \mathrm{C} 11$ antibody representing sAPPFL and sAPP95 in cell-free conditioned media from control GFP-transfected HEKswe incubated at $37^{\circ} \mathrm{C}$ up to 18 hours (1080 minutes) with the recombinant MT5-MMP catalytic domain (cdMT5). The right lane represents a control of sAPPFL and sAPP95 production obtained from the supernatants of HEKswe cells transfected with MT5. Note that only sAPPFL is naturally enriched in GFP-transfected HEKswe and that cdMT5 was unable to generate sAPP95 in vitro. Values are the mean \pm SEM of at least four independent cultures. $* P<.05$ and *** $P<.001$ vs GFP untreated group. ANOVA followed by post hoc Fisher's LSD test (C) and Student's $t$ test (D-I)
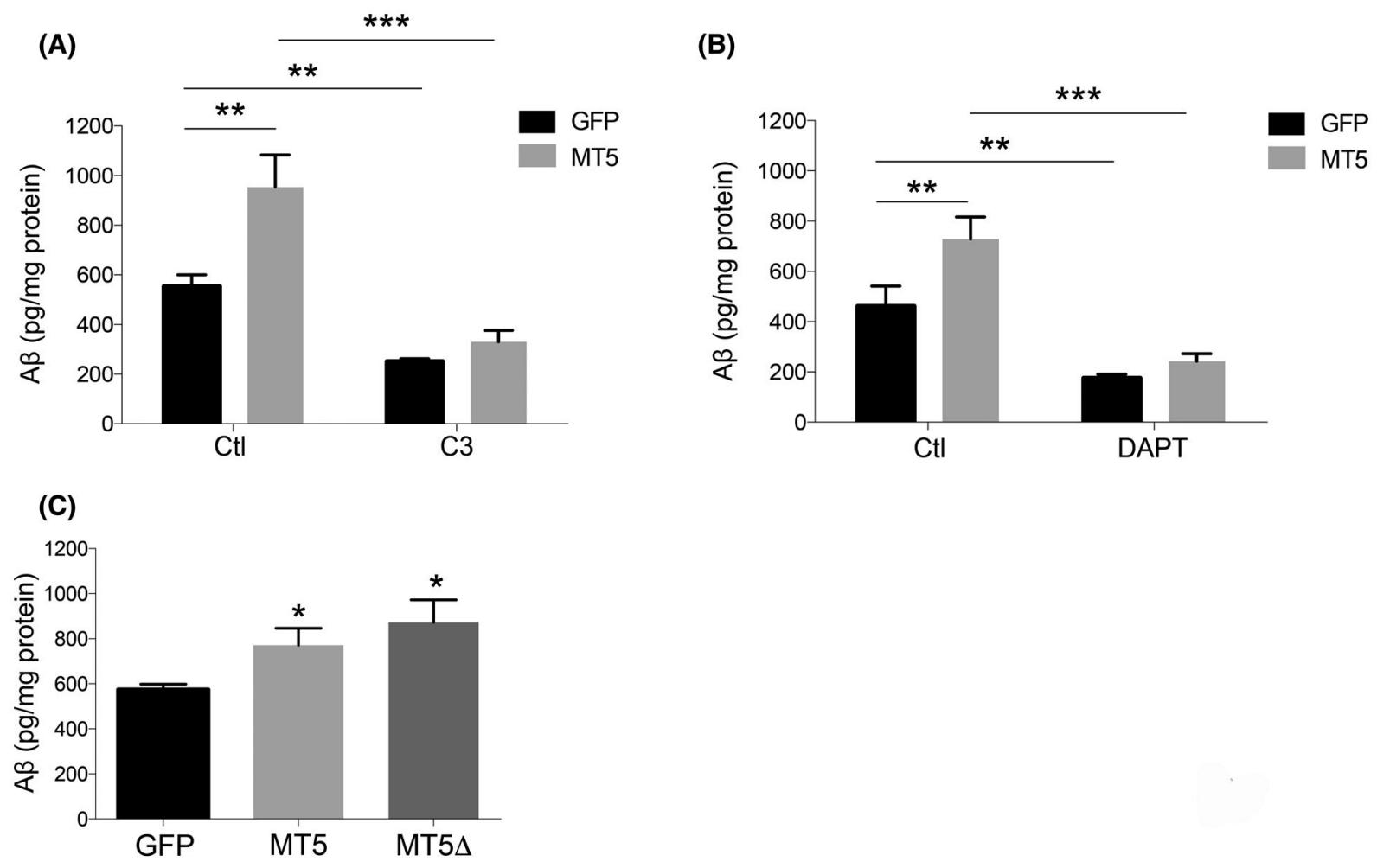

F I G URE $2 \beta$ - and $\gamma$-secretase mediate the upregulation of A $\beta$ levels by MT5-MMP and inactive MT5-MMP is also pro-amyloidogenic. A, B, Histograms showing ELISA quantification of A $\beta 40$ levels (pg/mg protein) in the supernatants of HEKswe cultures $(n=4-6) 48$ hours after transfection with GFP control and active MT5-MMP/GFP (MT5) in the presence or not of $10 \mu \mathrm{M}$ of C3 (A) and DAPT (B). C, Histogram showing ELISA quantification of A $\beta 40$ levels (pg/mg protein) in the supernatants of HEKswe cultures ( $\mathrm{n}=4-9) 48$ hours after transfection with GFP control, active (MT5) or inactive (MT5 $\Delta$ ) MT5-MMP/GFP constructs. Note that MT5 $\Delta$ is as effective as MT5 in stimulating A $\beta$ accumulation. Values are the mean of the indicated independent cultures. $* P<.05$, $* * P<.01$, and $* * * P<.001$ vs GFP untreated group. ANOVA followed by post hoc Fisher's LSD test

(PSENEN), and Aph1a (APH1A). We also investigated possible changes in the expression of genes encoding $\mathrm{A} \beta$ degrading enzymes such as $M M P 2, M M P 9, M M E$ (neprilysin), and CTSD (cathepsin D). We found no significant changes for all genes, except for the expected increase in $M M P 24$ levels after transfections of the active and inactive variants (Table 2).
The above data ruled out a possible transcriptional role of MT5-MMP, suggesting that proteolysis was responsible for MT5-MMP-mediated effects. However, both active (MT5) and inactive (MT5 $\Delta$ ) variants significantly increased the extracellular levels of A $\beta 40$ (Figure $2 \mathrm{C}$ ), indicating that the proamyloidogenic effects of MT5-MMP could also depend on non-proteolytic activities. This was consistent with previous 


\begin{tabular}{|c|c|c|c|c|}
\hline \multirow[b]{2}{*}{ Genes } & \multicolumn{3}{|l|}{ Mean $( \pm$ SEM) } & \multirow[b]{2}{*}{ Statistics } \\
\hline & GFP & MT5 & MT5 & \\
\hline$A P P$ & $1.01( \pm 0.02)$ & $0.971( \pm 0.07)$ & $0.933( \pm 0.06)$ & ns \\
\hline$M M P 2$ & $1.06( \pm 0.21)$ & $1.091( \pm 0.14)$ & $1.31( \pm 0.16)$ & ns \\
\hline$M M P 9$ & $1.071( \pm 0.23)$ & $0.916( \pm 0.12)$ & $1.086( \pm 0.09)$ & ns \\
\hline MMP14 & $1.043( \pm 0.15)$ & $1.413( \pm 0.2)$ & $0.814( \pm 0.12)$ & ns \\
\hline$M M P 24$ & $1.08( \pm 0.25)$ & $192.684( \pm 35.7)$ & $88.603( \pm 15.51)$ & $* * *$ \\
\hline TIMP1 & $1.095( \pm 0.22)$ & $0.974( \pm 0.07)$ & $1.248( \pm 0.18)$ & ns \\
\hline$M M E$ & $1.061( \pm 0.19)$ & $1.162( \pm 0.15)$ & $1.188( \pm 0.25)$ & ns \\
\hline$C T S D$ & $1.006( \pm 0.07)$ & $0.989( \pm 0.06)$ & $1.082( \pm 0.17)$ & ns \\
\hline$B A C E 1$ & $1.084( \pm 0.23)$ & $1.196( \pm 0.13)$ & $1.247( \pm 0.15)$ & ns \\
\hline NCTSN & $1.003( \pm 0.04)$ & $0.971( \pm 0.09)$ & $1.119( \pm 0.096)$ & ns \\
\hline PSENEN & $1.01( \pm 0.086)$ & $0.869( \pm 0.07)$ & $1.102( \pm 0.048)$ & ns \\
\hline APHIA & $1.059( \pm 0.18)$ & $0.997( \pm 0.24)$ & $1.106( \pm 0.14)$ & ns \\
\hline PSEN1 & $1.044( \pm 0.16)$ & $0.852( \pm 0.11)$ & $1.017( \pm 0.049)$ & ns \\
\hline PSEN2 & $1.040( \pm 0.16)$ & $0.926( \pm 0.13)$ & $1.341( \pm 0.17)$ & ns \\
\hline
\end{tabular}

TA B LE 2 MT5-MMP overexpression does not affect the expression of genes potentially involved in APP/A $\beta$ metabolism

Note: ANOVA followed by post hoc Fisher's LSD test.

Abbreviation: ns, no significance.

$* * * P<.001$ vs GFP.

data indicating that MT5-MMP could impact on APP metabolism by regulating its cellular traffic. ${ }^{17}$ In support of this idea, MT5 and MT5 $\Delta$ significantly increased the content of $\mathrm{APP} / \mathrm{A} \beta$ immunoreactivity in early endosomes compared to control, as shown by combined Alexa Fluor ${ }^{647}$-transferrin pulse labeling and immunofluorescence with the $6 \mathrm{E} 10$ antibody (Figure 3A). These results were further confirmed by increased co-localization of an antibody directed against the $\mathrm{C}$-terminal end of $\mathrm{A} \beta$ and the early endosome marker EEA1 (Figure 3B), while endosomes content remained stable (Figure S1A). Moreover, we tested the impact of MT5-MMP on the trafficking of $6 \mathrm{E} 10^{+}$APP fragments, including $\mathrm{A} \beta$, to the lysosomal degrading pathway. Interestingly, LAMP1 ${ }^{+}$ lysosomes from MT5 $\Delta$-expressing cells showed a $127 \%$ and $96 \%$ significant increase in $6 \mathrm{E} 10^{+}$immunoreactivity compared to GFP and MT5 groups, respectively (Figure 3C). Meanwhile, lysosome content increased only by $10 \%$ in MT5 $\Delta$ cells compared to GFP control (Figure S1B).

We deduce from these data that MT5-MMP could influence endo-lysosomal APP traffic to stimulate A $\beta$ production in concert with $\beta$ - and $\gamma$-secretases, and that non-catalytic MT5-MMP domains are likely involved in such a mechanism.

\section{3 | Differential impact of MT5-MMP domains on APP metabolism}

To determine the specific impact of MT5-MMP domains on APP metabolism, we designed plasmids constructs encoding full-length (FL) active (MT5 ${ }^{\mathrm{FL}}$ ) and inactive $\left(\mathrm{MT}^{\Delta}\right.$ ) MT5MMP, as well as truncated variants lacking functional proteinase domains as follows: catalytic (MT5 ${ }^{\mathrm{CAT}-}$ ), hemopexin $\left(\mathrm{MT}^{\mathrm{HPX}-}\right)$, extracellular (MT5 ${ }^{\mathrm{EXT}-}$ ), transmembrane and intracytoplasmic domains $\left(\mathrm{MT}^{\mathrm{TM} / \mathrm{IC}-}\right.$ ), and intracytoplasmic domain alone (MT5 ${ }^{\mathrm{IC}-}$ ), all N-terminally tagged with Flag (Figure 4A). After transient transfection in HEKswe cells (Figure 4B, top panel), none of the variants affected canonical intracellular full-length APP (APPFL) (Figure 4B, second panel and C) or sAPPFL (Figure 4B, third panel and D). As expected, MT5 ${ }^{\mathrm{FL}}$ generated sAPP95, while proteolytically inactive $\mathrm{MT}^{\Delta}{ }^{\Delta}$ MT5 $^{\mathrm{CAT}-}$, and MT5 ${ }^{\mathrm{EXT}-}$ did not (Figure 4B, third panel and E). However and most interestingly, the ability of MT5-MMP to produce sAPP95 was significantly reduced upon deletion of the non-catalytic domains MT5 ${ }^{\mathrm{HPX}-}$, $\mathrm{MT}^{\mathrm{TM} / \mathrm{IC}-}$, and $\mathrm{MT}^{\mathrm{IC}-}$ (Figure $4 \mathrm{~B}$, third panel and E).

We then tested how the expression of MT5-MMP variants that impaired the ability to process APP could affect $\mathrm{A} \beta$ levels. Among the truncated variants, only MT5 ${ }^{\text {EXT- }}$ significantly increased the extracellular $A \beta 40$ concentration measured by ELISA (Figure 4F), indicating that the C-terminal part of MT5-MMP alone could promote amyloidogenesis.

\section{4 | The C-terminal domain of MT5-MMP specifically affects C99 levels}

Based on previous observations showing that MT5-MMP deficiency in 5xFAD mice drastically reduce C99 levels, ${ }^{16}$ 


\section{(A)}

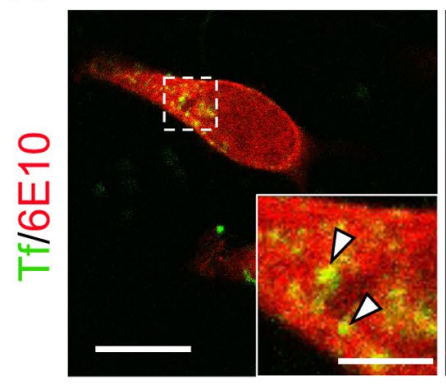

MT5

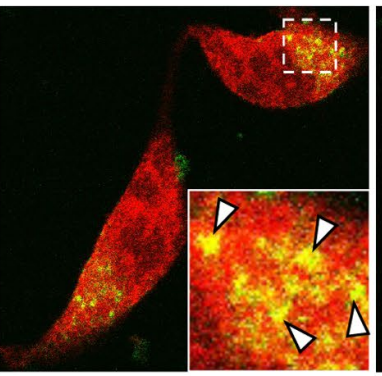

MT5 $\Delta$
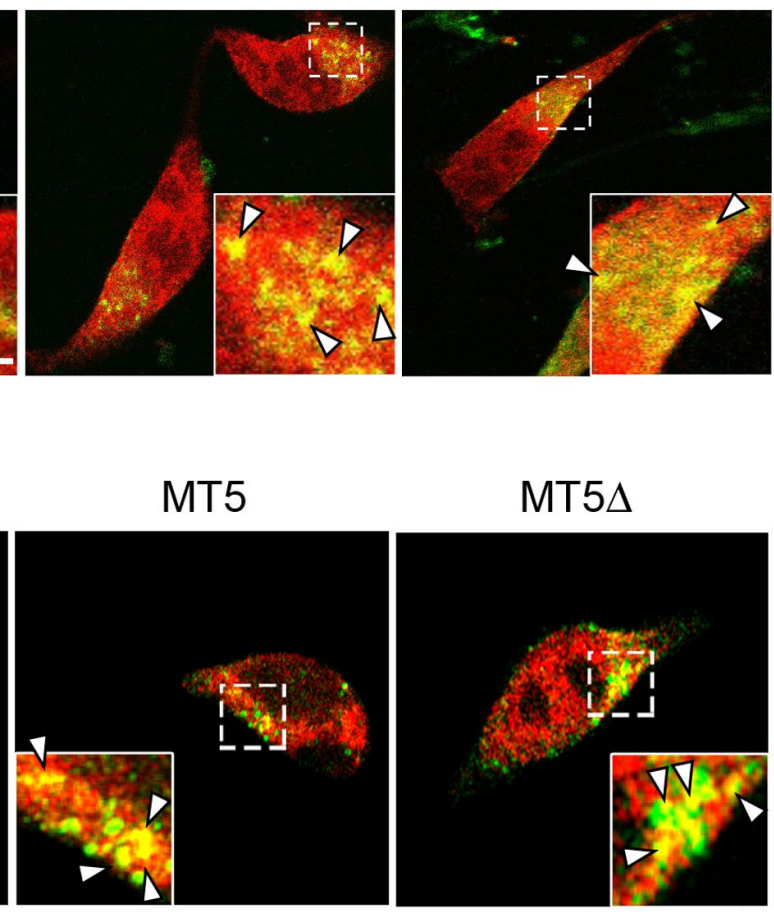

(B)

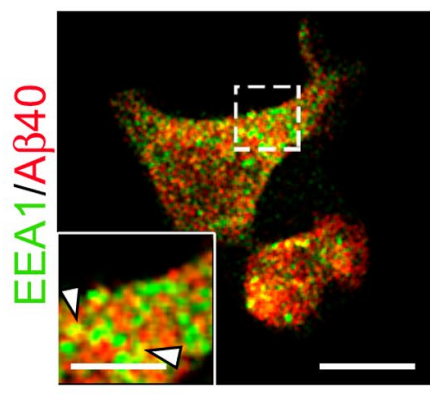

MT5 $\Delta$

MT5

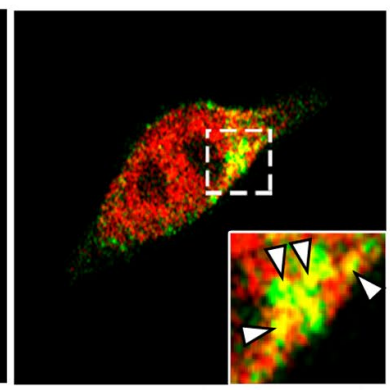

Tf/6E10
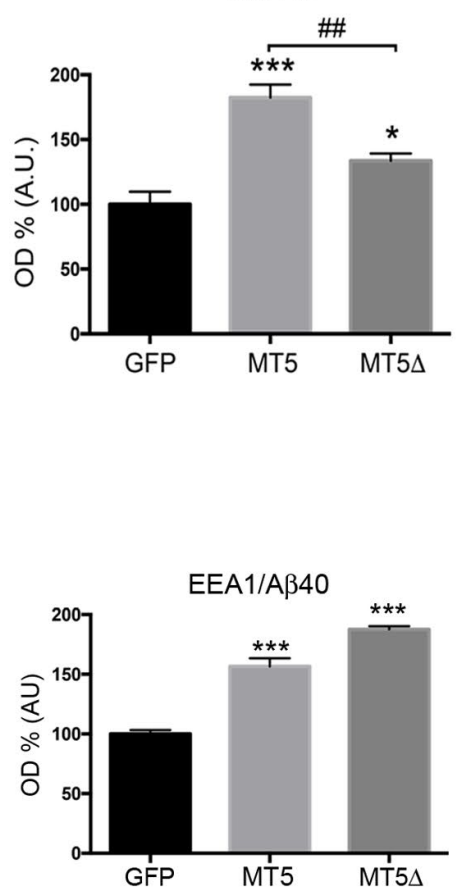

(C)

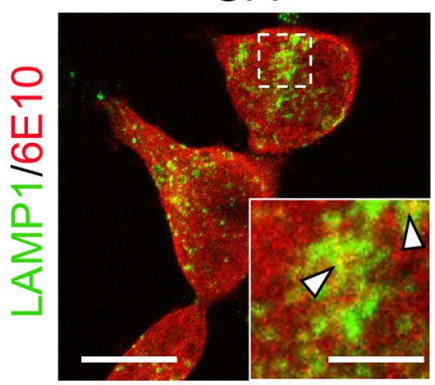

MT5

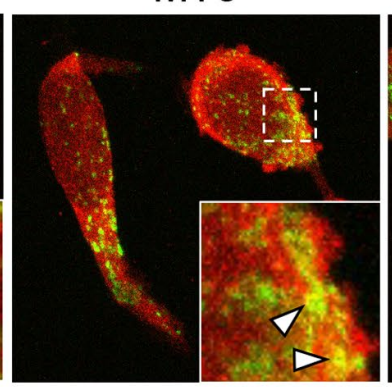

MT5 $\Delta$

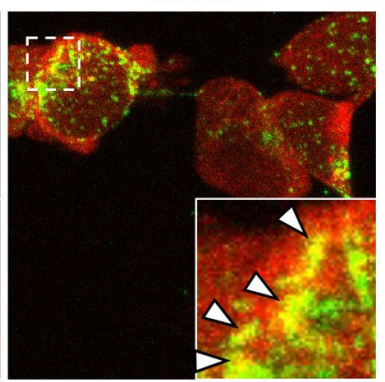

\section{LAMP-1/6E10}

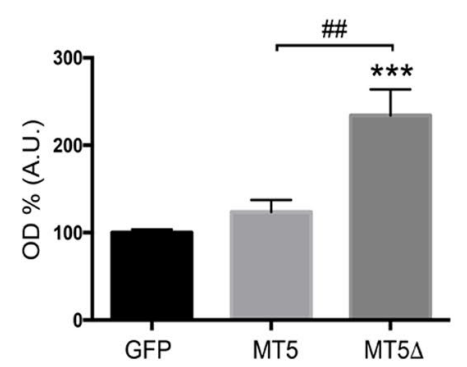

F I G U R E 3 MT5-MMP modulation promotes the distribution of APP/A $\beta$ in early endosomes and lysosomes. A, Single plane confocal micrographs showing anti-6E10 ${ }^{+}$immunoreactivity (red) in HEKswe cells incubated for 30 minutes at $37^{\circ} \mathrm{C}$ with transferrin-Alexa Fluor ${ }^{647}$ (green; Tf) to label early endosomes, 48 hours after transfection with constructs coding for GFP control, active MT5-MMP/GFP (MT5) or inactive MT5 $\Delta$ MMP/GFP $(\mathrm{MT} 5 \Delta)$. Arrowheads in the inset illustrate areas of colocalization between $6 \mathrm{E} 10^{+}$and $\mathrm{Tf}^{+}$signals (yellow). The histogram on the right shows the $\%$ of colocalization in arbitrary units (AU) between $6 \mathrm{E} 10^{+}$and $\mathrm{Tf}^{+}$with respect to GFP control. B, Single plane confocal micrographs showing anti-A $\beta 40$ immunoreactivity (red) and anti-EEA1 immunoreactivity (green) to label early endosomes in HEKswe cells 48 hours after transfection with GFP, MT5, or MT5 $\Delta$ constructs. Arrowheads in the inset illustrate areas of colocalization between A $\beta 40^{+}$and EEA $1^{+}$signals (yellow). The histogram on the right shows the $\%$ of colocalization between A $\beta 40^{+}$and EEA $1^{+}$signals in HEKswe cells transfected with GFP, MT5, or MT5 $\Delta$ constructs. C, Single plane confocal micrographs showing anti-6E10 (red) and anti-LAMP-1 immunoreactivity (green) in HEKswe cells 48 hours after transfection with either GFP, MT5, or inactive MT5 $\Delta$ constructs. Arrowheads in the inset illustrate areas of colocalization between $6 \mathrm{E} 10^{+}$and LAMP- $1^{+}$signals (yellow). The histogram on the right shows the $\%$ of colocalization between $6 \mathrm{E} 10^{+}$and LAMP- $1^{+}$signals with respect to GFP control. Values represent the mean \pm SEM of four independent cultures. $* * P<.01$ and $* * * P<.01$ vs GFP. ${ }^{\# \#} P<.01$ vs MT5. ANOVA followed by post hoc Fisher's LSD test. Scale bars $10 \mu \mathrm{m}$. Scale bar in the insets, $3 \mu \mathrm{m}$

we next investigated the possibility that different MT5-MMP domains could affect $\mathrm{C} 99$, with the additional interest that C99 is increasingly considered a relevant neurotoxic factor in $\mathrm{AD} .{ }^{6,7,9,10,36}$ To test the specific impact of MT5-MMP domains on C99, we first mimicked AD-like C99 accumulation by transiently overexpressing a C99 construct in HEK cells. After transfection of the plasmid encoding C99, the APP-CTF antibody revealed a $\sim 13 \mathrm{kDa}$ immunoreactive band matching the predicted molecular weight of C99 (Figure 5A). A second band of $\sim 11 \mathrm{kDa}$ (Figure 5A, top panel) was abolished 
by the $\alpha$-secretase inhibitor GI254023X (not shown) and was not detected by the $6 \mathrm{E} 10$ antibody that recognizes an epitope in the $\mathrm{N}$-terminal portion of $\mathrm{A} \beta$ upstream the $\alpha$-cleavage site (Figure 5A, middle panel). Therefore, we concluded that the $\sim 11 \mathrm{kDa}$ band represented $\mathrm{C} 83$ resulting from the

(A)

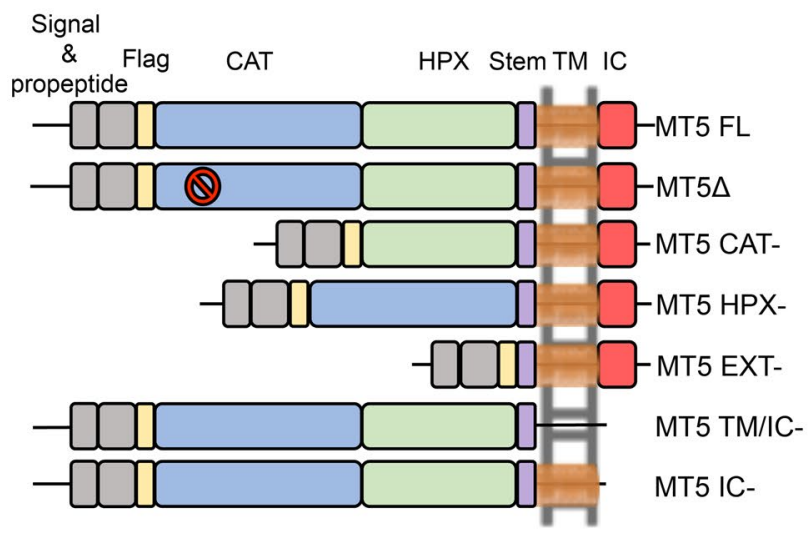

(C)
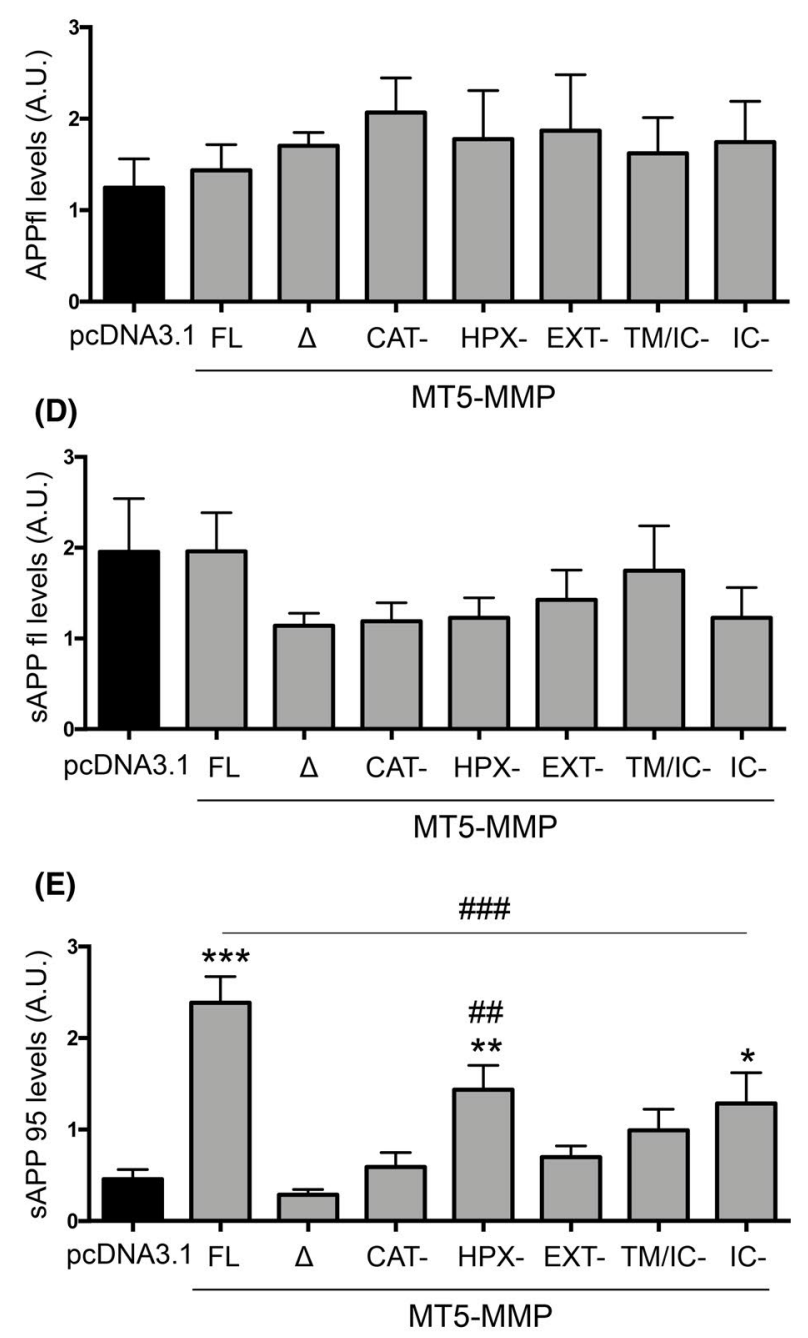

cleavage of C99 by $\alpha$-secretase, as previously observed. ${ }^{25}$ It has to be noted that co-transfection of C99 and the MT5MMP variants had no effect on cell viability as measured by the MTT test (Figure S2A), probably indicating that $A \beta$ in the low $\mathrm{nM}$ range was not sufficient to induce toxicity

(B)

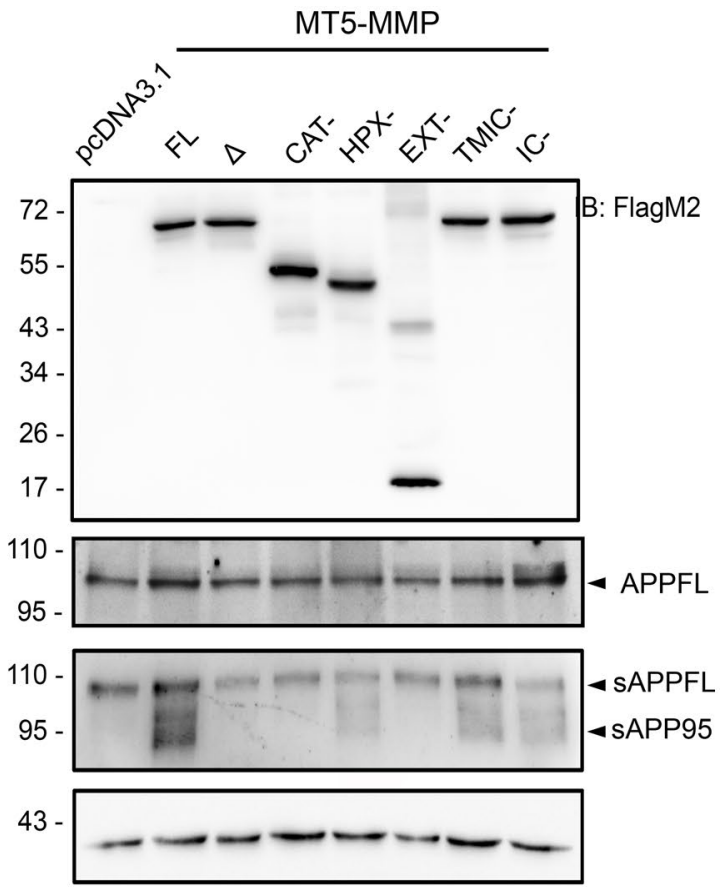

(F)

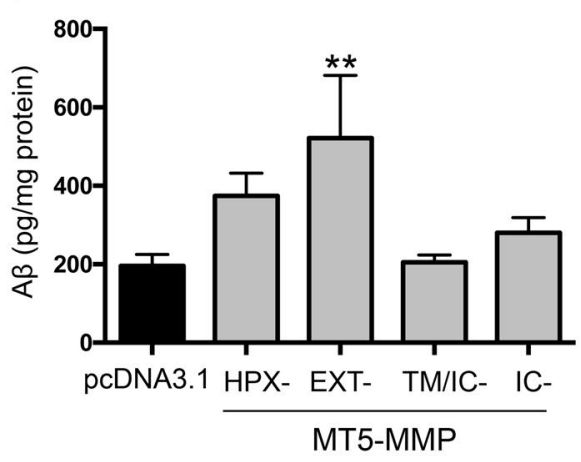


F I G U R E 4 MT5-MMP domains differently affect sAPP95 and A $\beta$ production. A, Schematic representation of different MT5-MMP variants tagged with Flag on the N-ter, including full-length active (MT5 FL) and inactive (MT5 $\Delta$ ) MT5-MMP, as well as truncated MT5-MMP variants without catalytic $\left(\mathrm{CAT}^{-}\right)$, hemopexin $\left(\mathrm{HPX}^{-}\right)$, extracellular $\left(\mathrm{EXT}^{-}\right)$, transmembrane/intracytoplasmic $\left(\mathrm{TM} / \mathrm{IC}^{-}\right)$, and intracytoplasmic $\left(\mathrm{IC}^{-}\right)$ domains. B, WB representing these MT5-MMP variants and the pcDNA3.1 control plasmid 48 hours after transient transfection of HEKswe cells, using an anti-FlagM2 antibody (first panel). Total full-length APP (APPFL) from cell lysates (second panel) and soluble APP fragments sAPPFL and sAPP95 in cell supernatants (third panel), using the anti-APP 22C11 antibody. Actin-loading controls are represented in the fourth panel. C-E, Histograms showing actin-normalized quantifications of APPFL in cell lysates (C), sAPPFL (D), and sAPP95 (E) in cell supernatants. F, Histogram showing ELISA quantification of A 340 levels ( $\mathrm{pg} / \mathrm{mg}$ protein) in the supernatants of HEKswe cultures 48 hours after transfection with pcDNA3.1, $\mathrm{HPX}^{-}, \mathrm{EXT}^{-}, \mathrm{TM} / \mathrm{IC}^{-}$, and $\mathrm{IC}^{-}$constructs. Note that only EXT ${ }^{-}$increases A $\beta$ levels with respect to pcDNA3.1, TM/IC ${ }^{-}$, and $\mathrm{IC}^{-}$groups. Values represent the mean \pm SEM of five independent cultures. ${ }^{*} P<.05$, $* * P<.01$, and $* * * P<.005$ vs pcDNA3.1. ${ }^{\# \#} P<.01$ and ${ }^{\# \# \#} P<.001$ vs MT5 FL. ANOVA followed by post hoc Fisher's LSD test

in HEK cells. Although the levels of C99 were not measured, these might not be high enough to achieve toxicity in HEK cells. Alternatively, the relatively short period of C99 accumulation could also explain the lack of toxicity. These hypothesis are based on previous work showing that C99 is capable to induce toxic effects in a number of experimental settings. ${ }^{36}$ In these conditions, the MT5 ${ }^{\mathrm{IC}-}$ variant caused a striking reduction of $\mathrm{C} 99$ levels, which was even more pronounced in MT5 ${ }^{\text {TM/IC- }}$ expressing cells (Figure 5A-C). In contrast, the expression of MT5 $5^{\mathrm{HPX}-}$ and $\mathrm{MT} 5^{\mathrm{EXT}-}$ significantly increased C99 levels when evaluated with the 6E10 antibody (Figure 5A-C). No difference in C99 levels was observed after transfection of MT5 ${ }^{\mathrm{FL}}, \mathrm{MT}^{\Delta}$, and MT5 ${ }^{\mathrm{CAT}-}$. Noticeably, all the MT5-MMP variants except MT5 ${ }^{\mathrm{HPX}-}$ nearly abolished C83 levels (Figure 5A,D). ELISA revealed a significant reduction of more than $50 \%$ in $\mathrm{A} \beta 40$ content after expression of MT5 $5^{\mathrm{TM} / \mathrm{IC}-}$ and MT5 ${ }^{\mathrm{IC}-}$ (Figure $5 \mathrm{E}$ ), ruling out the possibility that the drop in $\mathrm{C} 99$ caused by these variants was due to an increased conversion of $C 99$ to $A \beta$. Of note, no differences in $\mathrm{A} \beta$ levels were observed after expression of MT5 ${ }^{\mathrm{HPX}-}$ and MT5 ${ }^{\mathrm{EXT}-}$, suggesting that their apparent cumulative effects on $\mathrm{C} 99$ did not impact $\mathrm{A} \beta$ production.

\section{5 | The C-terminal domains of MT5- MMP and MT1-MMP have opposite effects}

We investigated next whether the striking drop in C99 caused by the deletion of the C-terminal domains of MT5-MMP was specific for this MMP. To this end, we used for comparison the close homolog MT1-MMP, whose active fulllength $\left(\mathrm{MT}^{\mathrm{FL}}\right)$, inactive full-length $\left(\mathrm{MT}^{\Delta}{ }^{\Delta}\right)$ or IC-deleted $\left(\mathrm{MT}^{\mathrm{IC}-}{ }^{-}\right.$) variants were transiently co-expressed with $\mathrm{C} 99$ (Figure 6A). ${ }^{24}$ Using the APP-CTF antibody (Figure 6B), we found that $\mathrm{MT}^{\mathrm{FL}}$ and $\mathrm{MT}^{\Delta}{ }^{\Delta}$ variants did not affect $\mathrm{C} 99$ content compared to pcDNA3.1. On the contrary, $\mathrm{MT}^{\mathrm{IC}-}$ significantly increased by $159 \%$ the levels of C99 (Figure 6B,C) and by $207 \%$ those of C 83 (Figure 6B,D). These data were in clear contrast with the strong reduction of C99 and C83 levels induced by MT5 ${ }^{\text {IC- }}$ (Figure 5C,D), arguing in favor of specific roles of the intracellular domains of MT5-MMP in the control of APP CTF levels.

\section{6 | The C-terminal part of MT5-MMP interacts with C99}

Given the major effects on C99 levels caused by the modulation of the TM and IC domains of MT5-MMP, as well as our previous results showing an interaction of MT5-MMP and $\mathrm{APP},{ }^{16}$ we searched for specific interactions of the Cterminal portion of MT5-MMP that might underlie its effects on C99. Accordingly, we conducted co-immunoprecipitation experiments in HEK cells after co-transfection of Flag $\mathrm{MT5}^{\mathrm{FL}}$ or MT5 ${ }^{\mathrm{EXT}-}$ with a HA-C99 construct that allowed us to distinguish transfected C99 from endogenous APP species. HA-C99 (Figure 7A, top panel) and MT5-MMP variants (Figure 7A, middle panel) were readily expressed after transfection. C83 was not detected in this experiment with the anti-HA antibody because the N-terminal HA tag was lost upon cleavage of C99 by $\alpha$-secretase. FlagM2 immunoprecipitated complexes were immunoreactive for the HA antibody, indicating that the C-terminal domain of MT5MMP interacts with C99 (Figure 7B). The use of unspecific IgG demonstrated lack of immunoprecipitation of MT5 $5^{\text {EXT- }}$ (Figure 7B). Reverse immunoprecipitation with the anti-HA antibody followed by WB with an anti-flagM2 antibody revealed that $\mathrm{MT}^{\mathrm{EXT}-}$ and $\mathrm{MT}^{\mathrm{FL}}$ were also immunoprecipitated (Figure 7C). Taken together, these data showed that TM and/or IC domains of MT5-MMP could mediate C99 interactions.

\section{7 | The C-terminal part of MT5-MMP promotes $\mathrm{C} 99$ endosomal sorting}

We wanted next to gain insight into the cellular distribution of HA-C99 after co-transfections with different MT5-MMP variants. We first confirmed that the inclusion of HA did not interfere with the effects of the variants on C99. As illustrated in Figure 8A, MT5 ${ }^{\mathrm{TM} / \mathrm{IC}-}$ reduced the levels of HA-C99 and C83 in comparable manner to non-tagged C99 (Figure 5AC). Immunocytochemistry after co-transfection of HA-C99 with either pcDNA3.1, MT5 ${ }^{\mathrm{FL}}$, MT5 $5^{\mathrm{EXT}-}$, or MT5 $5^{\mathrm{TM} / \mathrm{IC}-}$ (Figure 8A-F) indicated that MT5 ${ }^{\text {EXT- }}$ significantly increased the localization of HA-C99 with early endosomal marker 
(A)

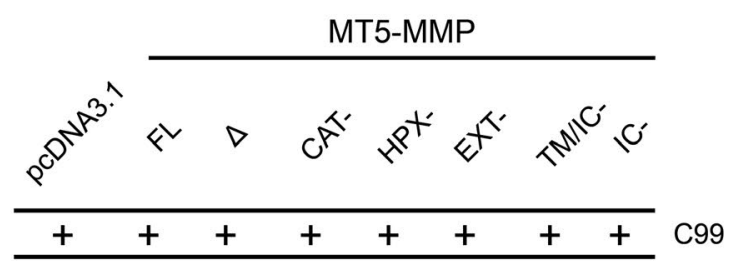

14,2

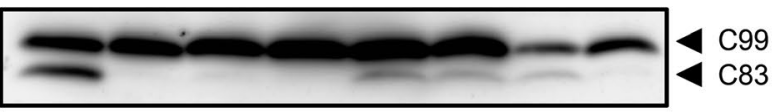

IB: APP-CTF

Lysates

14,2

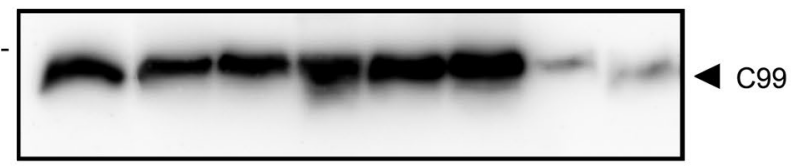

IB: 6E10

Lysates

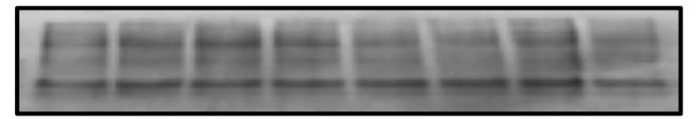

Ponceau

(D)

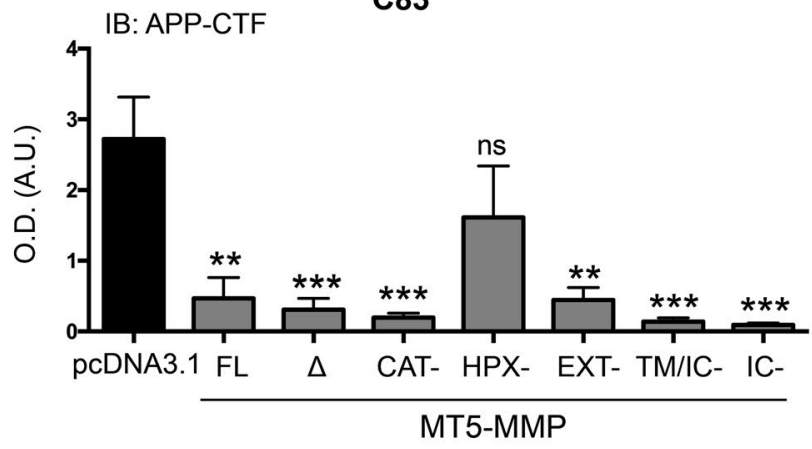

(B)

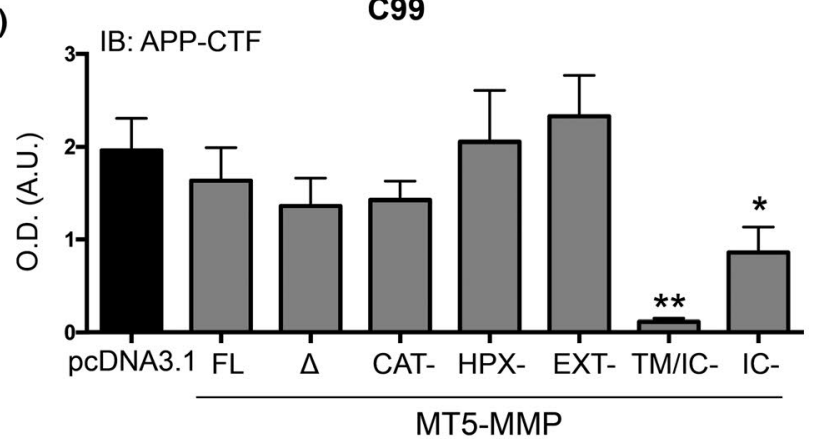

(C)

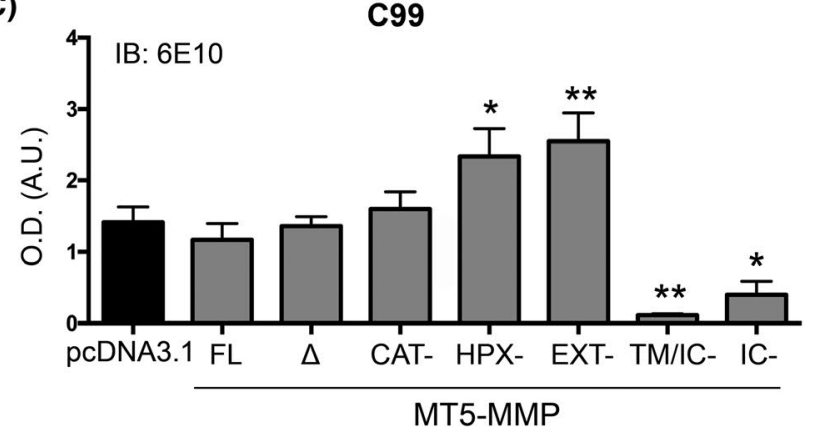

(E)

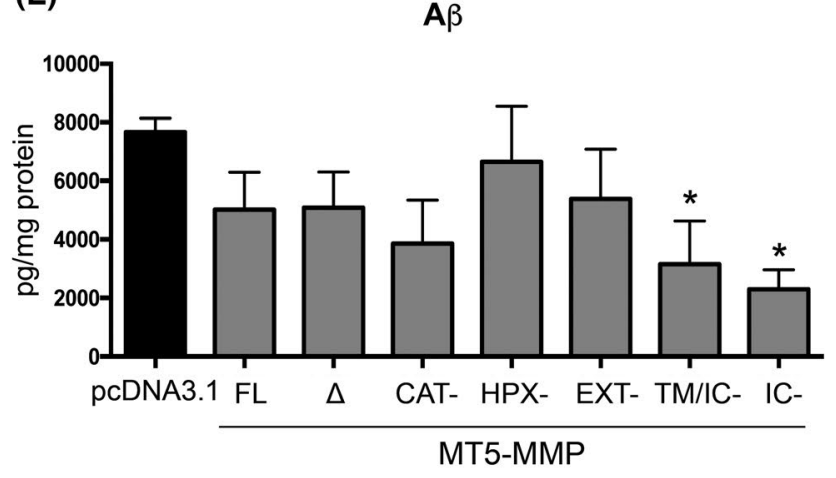

F I G U R E 5 MT5-MMP domains differently affect C99 stability. A, Representative WB of C99 and C83 levels in HEK cells 48 hours after co-transfection with C99 and either a control pcDNA3.1 plasmid and full-length (FL) active or inactive ( $\Delta$ ) MT5-MMP or MT5-MMP without catalytic $\left(\mathrm{CAT}^{-}\right)$, hemopexin $\left(\mathrm{HPX}^{-}\right)$, extracellular $\left(\mathrm{EXT}^{-}\right)$, transmembrane/intracytoplasmic $\left(\mathrm{TM} / \mathrm{IC}^{-}\right)$, and intracytoplasmic $\left(\mathrm{IC}^{-}\right)$domains, using APP-CTF antibody (top panel) and 6E10 antibody (middle panel). The bottom panel represents the protein loading controls with ponceau S staining. B-D, Histograms represent ponceau-normalized quantifications of C99 detected with both the APP-CTF (B) and 6E10 (C) antibodies, and C83 detected with APP-CTF antibody (D). E, Histogram showing ELISA quantification of A $\beta 40$ levels (pg/mg protein) in the supernatants of HEK cells $(\mathrm{n}=4) 48$ hours after co-transfection of C99 with either pcDNA3.1, FL, $\Delta, \mathrm{CAT}^{-}, \mathrm{HPX}^{-}$, EXT ${ }^{-}, \mathrm{TM}^{-} \mathrm{IC}^{-}$, and IC ${ }^{-}$. Values are the mean \pm SEM of four independent cultures. $* P<.05$, $* * P<.01$, and $* * * P<.001$ vs pcDNA3.1. ANOVA followed by post hoc Fisher's LSD test. ns: no significant

EEA1 (Figure 8B-F), while MT5 ${ }^{\mathrm{FL}}$ and MT5 $5^{\mathrm{TM} / \mathrm{IC}-}$ did not. All experimental groups exhibited equivalent EEA $1^{+}$signal, implying that MT5-MMP variants did not alter endosome content (Figure S2B). Overall, these data further suggest that the C-terminal domains of MT5-MMP modulate the stability and cellular distribution of C99, in particular upon deletion of the extracellular domain of the proteinase.

\section{8 | The control of C99 and C83 fate by MT5-MMP involves different cell scavenging systems}

The concomitant reduction of $\mathrm{C} 99$ and $\mathrm{A} \beta$ levels in cells expressing MT5 ${ }^{\mathrm{TM} / \mathrm{IC}-}$ and MT5 ${ }^{\mathrm{IC}-}$ (Figure 5) suggests that C99 is degraded by one or several scavenging systems. In 


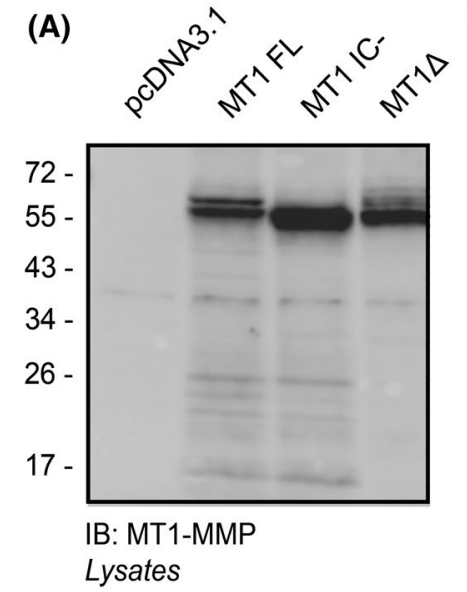

(C)

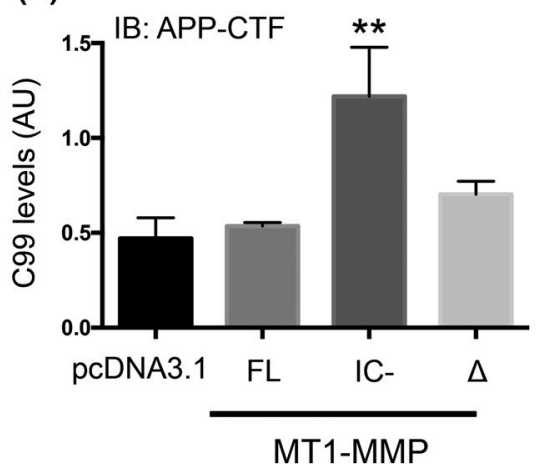

(B)
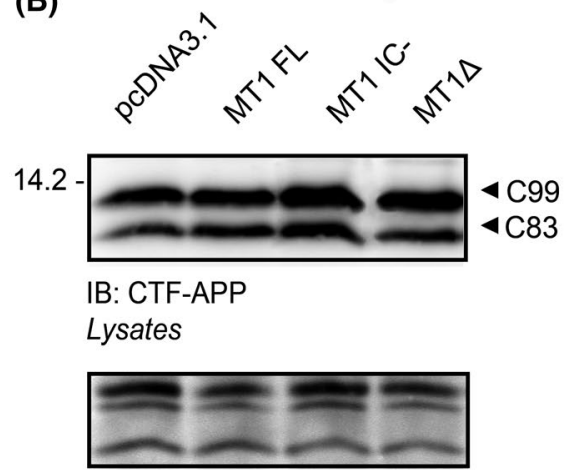

Ponceau

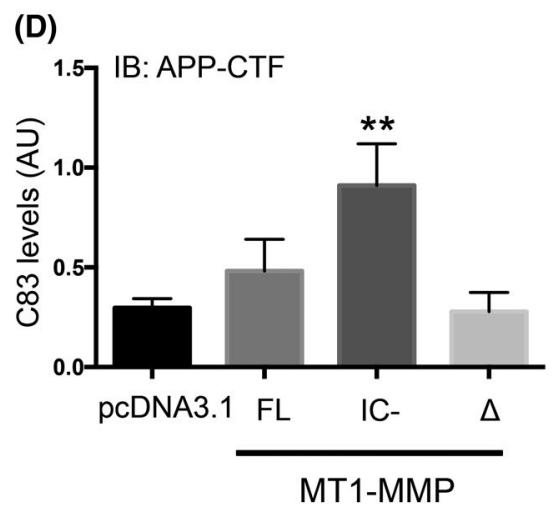

F I G U RE 6 Deleting the intracellular domain of MT1-MMP promotes the accumulation of C99 and C83. A, Representative WB of MT1-MMP immunoreactivity in HEK cells revealed by an anti-MT1-MMP antibody 48 hours after transfection of pcDNA3.1, MT1-MMP full-length (FL), MT1-MMP lacking the intracytoplasmic domain $\left(\mathrm{IC}^{-}\right)$, and inactive MT1-MMP (MT1 $\Delta$ ). B, WB representing C99 and C83 immunoreactivity using an APP-CTF antibody and below the protein loading controls with ponceau S staining. C, D, Ponceau-normalized quantifications of C99 (C) and C83 (D). Values are the mean \pm SEM of four independent cultures. $* * P<.01$ vs pcDNA3.1. ANOVA followed by post hoc Fisher's LSD test

order to further explore this possibility, cells co-expressing C99 with either control pcDNA3.1 or MT5-MMP variants were incubated with inhibitors of proteolytic systems potentially capable of degrading C99. We used DAPT, bafilomycin A1 (bafilomycin), and MG132, which, respectively, inhibit the $\gamma$-secretase, autophagolysosomal, and proteasome activities. Using the APP-CTF antibody on homogenates of cells co-transfected with pcDNA3.1 and C99, we observed that only MG132 induced a significant $66 \%$ accumulation of C99 compared to DMSO control, while DAPT and bafilomycin had no effect (Figure 9A-E). Therefore, in the absence of MT5-MMP, the excess of C99 in HEK cells was primarily eliminated by the proteasome and not by $\gamma$-secretase or autophagolysosomal activities. In contrast with C99, C83 was scavenged by the proteasome and also by $\gamma$-secretase. In pcDNA3.1-transfected cells, DAPT and MG132, respectively, increased C83 levels by $104 \%$ and $83 \%$, while bafilomycin had no effect (Figure 9A-D,F).

The expression of MT5-MMP variants deeply affected C99 levels depending on the pharmacological treatments
(Figure 9A-D,G-J). The most remarkable observation in control conditions was the drastic decrease of C99 content after the expression of $\mathrm{MT}^{\mathrm{TM} / \mathrm{IC}-}(80 \%)$ and $\mathrm{MT} 5^{\mathrm{IC}-}$ (64\%) compared to pcDNA3.1 (Figure 9A,G). Likewise, the drop in $\mathrm{C} 99$ levels in MT5 $5^{\mathrm{TM} / \mathrm{IC}-}$ and $\mathrm{MT}^{\mathrm{IC}-}$ cells was also highly significant compared to the $\mathrm{MT}^{\mathrm{FL}}$ and $\mathrm{MT}^{\text {EXT- }}$ groups. Interestingly, MT5 ${ }^{\text {EXT- }}$ expressing cells exhibited 50\% higher levels of C99 than $\mathrm{MT}^{\mathrm{FL}}$ under DMSO (Figure 9A,G), and such increase reached 229\% after DAPT treatment (Figure 9A,H). The increase was even bigger when comparing MT5 $5^{\text {EXT- }}$ to MT5 ${ }^{\mathrm{TM} / \mathrm{IC}-}$ expressing cells $(512 \%)$ and $\mathrm{MT}^{\mathrm{IC}-}(366 \%)$, as DAPT failed to rescue the drop of C99 levels in these groups (Figure 9A,H). Intriguingly, DAPT also accentuated the reduction in $\mathrm{MT}^{\mathrm{FL}}$ cells to $44 \%$ of pcDNA3.1 C99 values (Figure 9A,H). Bafilomycin significantly stimulated the accumulation of $\mathrm{C} 99$ in MT5 ${ }^{\text {EXT- }}$ cells with respect to pcDNA3.1 (41\%) and MT5 ${ }^{\mathrm{FL}}(256 \%)$. The effect of bafilomycin on the restoration of C99 levels in MT5 ${ }^{\mathrm{TM} / \mathrm{IC}-}$ was barely noticeable when compared with control cells. 

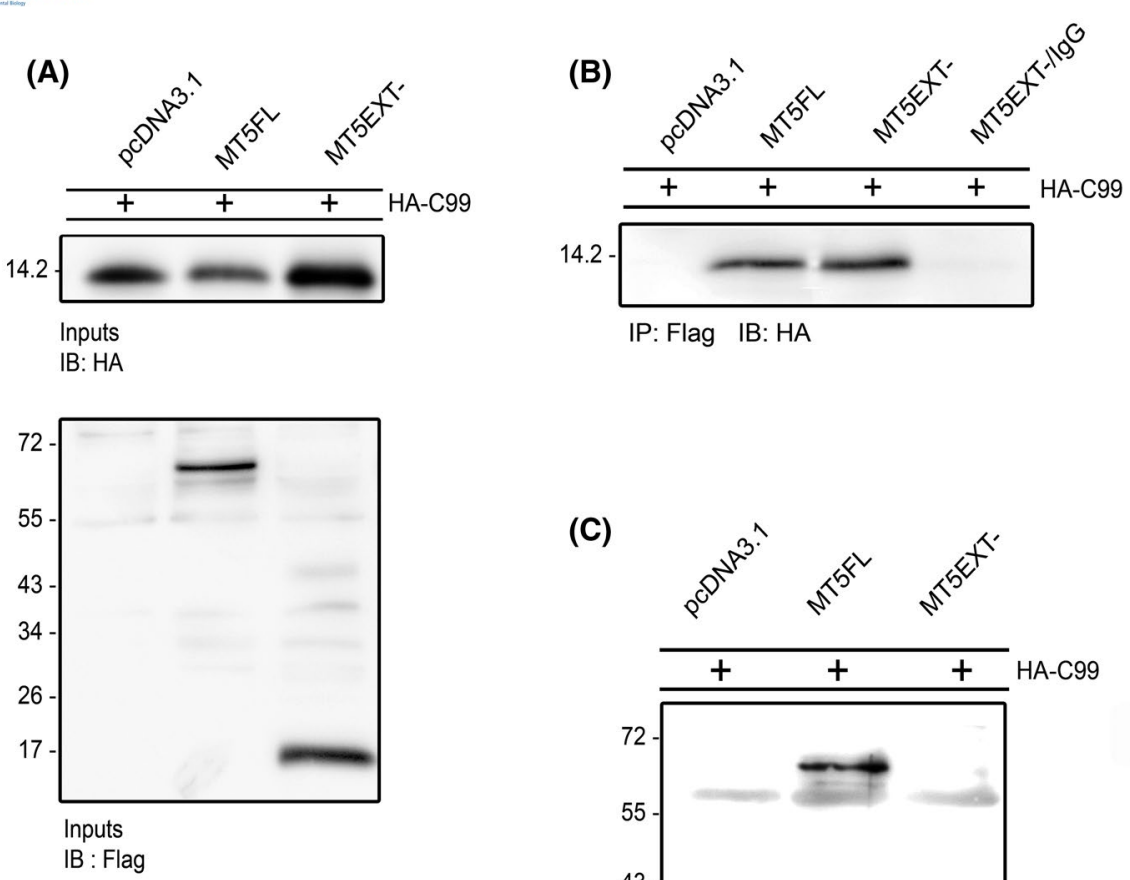

(C)

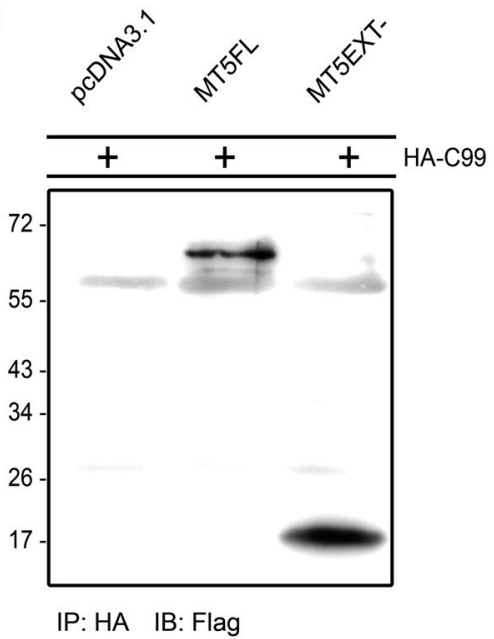

F I G U RE 7 The C-terminal domains of MT5-MMP mediate interactions with C99. A, WB showing anti-HA immunoreactivity in HEK cells 48 hours after co-transfection with HA-C99 and either pcDNA3.1 control, MT5 FL, and MT5 EXT-constructs (top panel). The middle panel shows representative anti-FlagM2 immunoreactivity for the transfected MT5-MMP variants (input), and the bottom panel, the protein loading controls with ponceau S staining. B, WB showing immunoprecipitation (IP) with a FlagM2 antibody or a non-specific IgG as negative control and immunoblot (IB) with an anti-HA antibody. C, WB showing reverse IP with an anti-HA antibody and IB with a FlagM2 antibody. Note that both MT5-MMP constructs immunoprecipitated HA-C99, suggesting the implication of the C-terminal domain of MT5-MMP in interactions with C99

Instead, bafilomycin brought C99 levels up to $69 \%$ of pcDNA3.1 in MT5 ${ }^{\text {TM/IC- }}$ cells (Figure 9C,I). In clear contrast with DAPT and bafilomycin, MG132 significantly restored the levels of C99 in MT5 ${ }^{\mathrm{TM} / \mathrm{IC}-}$ and MT5 ${ }^{\mathrm{IC}-}$ cells, which reached $59 \%$ and $74 \%$ of pcDNA3.1 values, respectively (Figure 9D,J). In the case of the MT5 ${ }^{\mathrm{TM} / \mathrm{IC}-}$ group, the difference was still statistically significant (Figure 9J). The widespread recovery of C99 levels under MG132 in most groups, together with an apparent lack of effect on MT5 $^{\text {EXT- }}$ cells, meant that C99 levels for this group were no longer different from those of any other experimental group (Figure 9J). Overall, the main conclusion is that C99 degradation caused by MT5 ${ }^{\mathrm{TM} / \mathrm{IC}-}$ and/or MT5 ${ }^{\mathrm{IC}-}$ variants is mainly driven by the proteasome, and to a lesser extent by the autophagolysosomal system, the latter being more efficient in controlling C99 in MT5 ${ }^{\text {EXT- }}$ cells.

All the MT5-MMP variants caused a drastic reduction of C83 levels in control conditions, ranking from $45 \%$ decrease in $\mathrm{MT}^{\mathrm{EXT}-}$ cells to $88 \%$ in MT5 ${ }^{\mathrm{TM} / \mathrm{IC}-}$ (Figure $9 \mathrm{~A}-\mathrm{D}, \mathrm{K}$ ). This is also illustrated in Figure 90, with the generalized decrease of the C83/C99 ratio in this group. DAPT restored
C83 levels back to pcDNA3.1 values in MT5 ${ }^{\text {EXT- }}$ cells. Moreover, DAPT rescued C83 in $\mathrm{MT} 5^{\mathrm{FL}}, \mathrm{MT}^{\mathrm{TM} / \mathrm{IC}-}$, and $\mathrm{MT}^{\mathrm{IC}-}$ around $55 \%$ of pcDNA3.1 values, clearly above the levels in control cells (Figure 9B,L). Figure 9P also illustrates the restoration of C83 by DAPT, as the drug leads to ratio values above 1 , except for the MT5 ${ }^{\text {EXT- }}$ group. The effect of bafilomycin on C83 was collectively less important than that of DAPT (Figure 9B,M). The main effect of bafilomycin was the restoration of $\mathrm{C} 83$ values in MT5 ${ }^{\text {EXT- }}$ cells compared to pcDNA3.1. In contrast, C83 levels in the other groups remained low (Figure 9A,C,K,M). Considering the $\mathrm{C} 83 / \mathrm{C} 99$ ratio, bafilomycin was the drug with the mildest effect (Figure 9Q). MG132 efficiently promoted C83 in cells transfected with pcDNA3.1 (Figure 9F), but not in those expressing any of the MT5-MMP variants. Their levels were collectively $\sim 80 \%$ lower compared to pcDNA3.1 and roughly unchanged compared to MT5-MMP variants in the DMSO control group (Figure 9A,D,K,N). This was further reflected in the C83/C99 ratio (Figure 9R). Overall, $\gamma$-secretase was very efficient in removing $\mathrm{C} 83$, and the effect was markedly accentuated in cells expressing the MT5 ${ }^{\text {EXT- }}$ variant. 
(A)

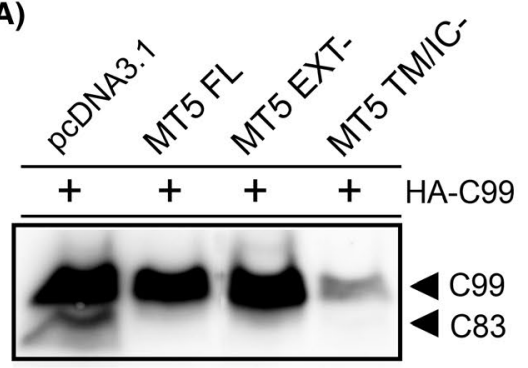

IB: APP-CTF

Lysates

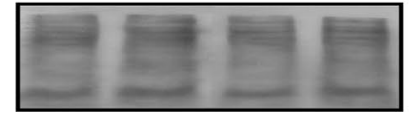

Ponceau
(B)
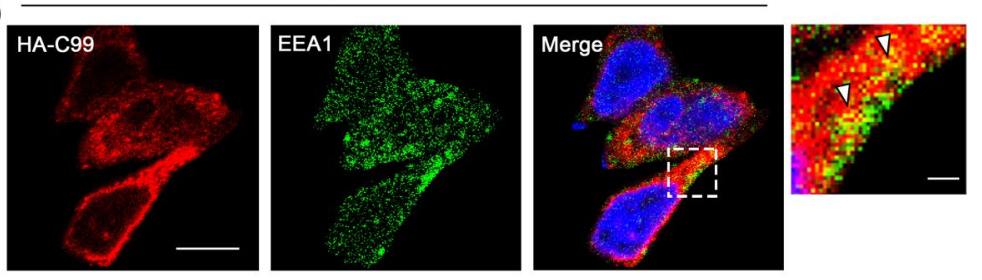

(C)

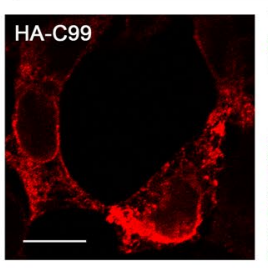

MT5 FL

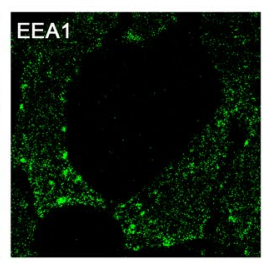

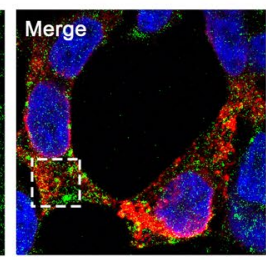

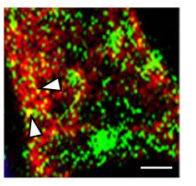

(D)

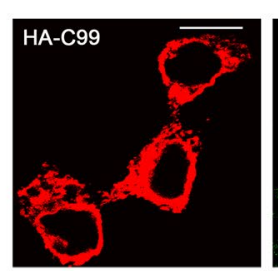

MT5 EXT-

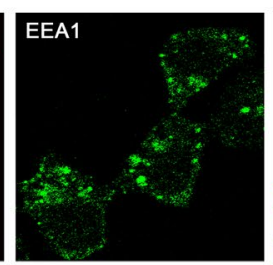

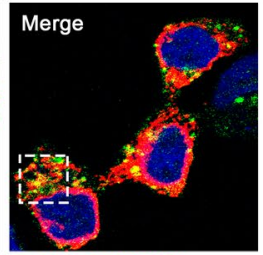

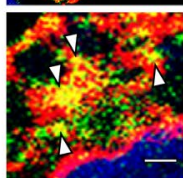

(E) MT5 TM/IC-

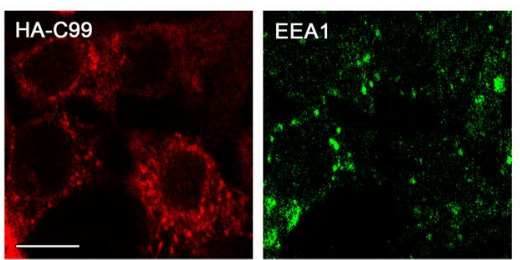

(F) HA/EEA1

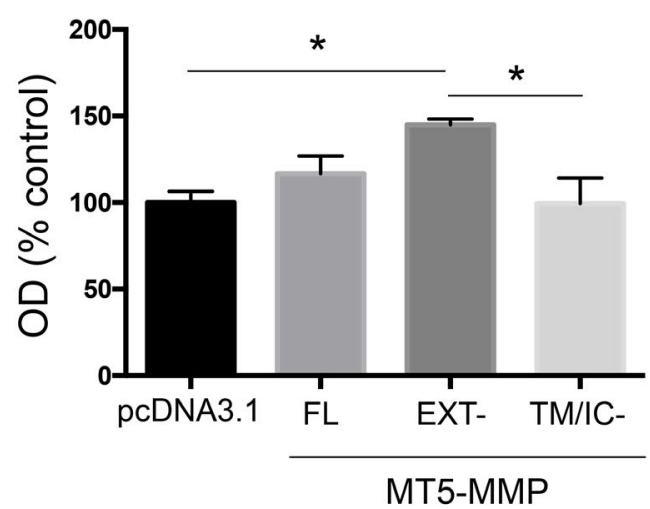

F I G U R E 8 MT5-MMP domains differently affect subcellular C99 localization. A, Representative WB of C99 and C83 levels in HEK cells 48 hours after co-transfection with HA-C99 and either control pcDNA3.1 plasmid, MT5-MMP full-length (MT5 FL) or MT5-MMP lacking the extracellular (MT5 EXT ${ }^{-}$) or transmembrane/intracytoplasmic (MT5 TM/IC ${ }^{-}$) domains, using an APP-CTF antibody. B-E, Single plane confocal micrographs showing immunofluorescence with anti-HA (red), early endosomal marker anti-EEA1 (green) an nuclei stained with Hoechst (blue) on HEK cells co-transfected for 16 hours with HA-C99 and pcDNA3.1 (B), MT5 FL (C), MT5 EXT ${ }^{-}$(D), or MT5 TM/IC ${ }^{-}$(E) constructs. Insets in the merge panel are shown as high-magnification images adjacent to the merge of $\mathrm{B}$ and $\mathrm{C}$ or below the merge of $\mathrm{D}$ and $\mathrm{E}$. White arrows in the insets indicate areas of colocalization between C99 and EEA1 (yellow). F, Histogram showing quantification of co-localization between EEA1 and HA-C99 referred as \% of pcDNA3.1 control. Only MT5 EXT ${ }^{-}$caused a significant increase in colocalization between HA-C99 ${ }^{+}$and EEA1 ${ }^{+}$ signals. Values represent the mean \pm SEM of four independent cultures. $* P<.05$, ANOVA followed by post hoc Fisher's LSD test. Scale bars, $10 \mu \mathrm{m}$. Scale bars in insets $2 \mu \mathrm{m}$ 


\section{4 | DISCUSSION}

Here we provide new evidence of MT5-MMP influence on APP metabolism, and A $\beta$ and $\mathrm{C} 99$ accumulation by both proteolysis-dependent and proteolysis-independent mechanisms. MT5-MMP C-terminal domains control the processing of APP into its soluble $95 \mathrm{kDa}$ form (sAPP95) in HEKswe. This mechanism does not involve endogenous MMP-2 or BACE1, although the latter is required for the pro-amyloidogenic effects of MT5-MMP. Interestingly, the full-length inactive form of MT5-MMP also induces the accumulation of $\mathrm{A} \beta$, while the $\mathrm{C}$-terminal non-catalytic
(A)

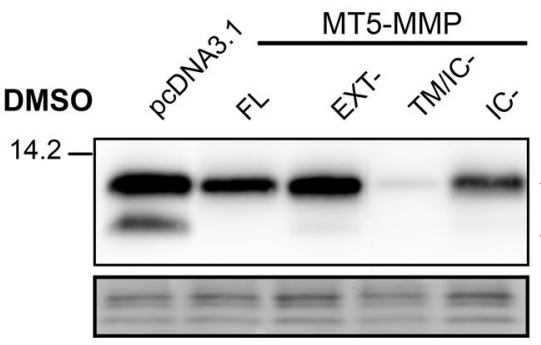

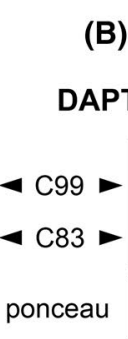

(B)

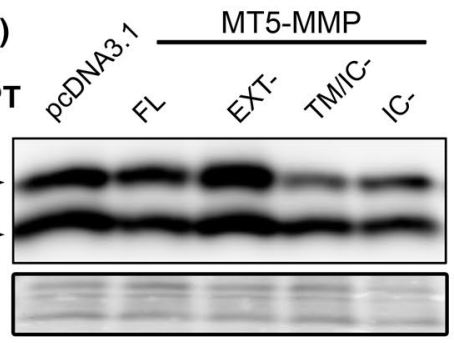
ponceau
IB: APP-CTF

(C)

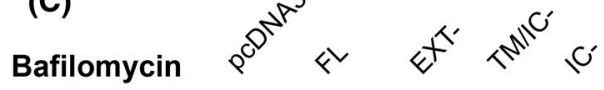

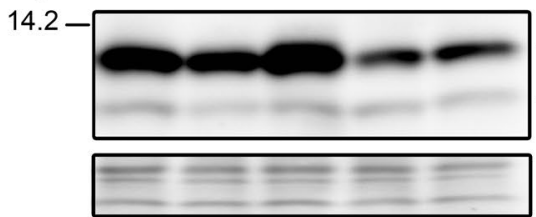

(D)

(D)

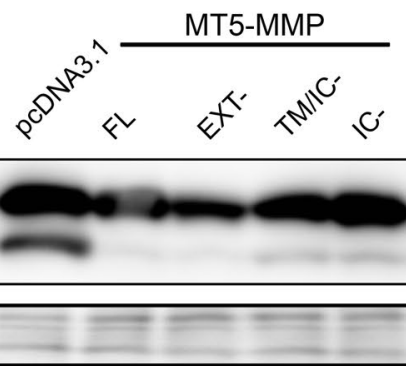

(E)

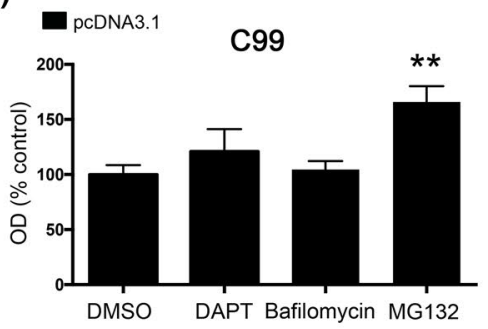

(F)

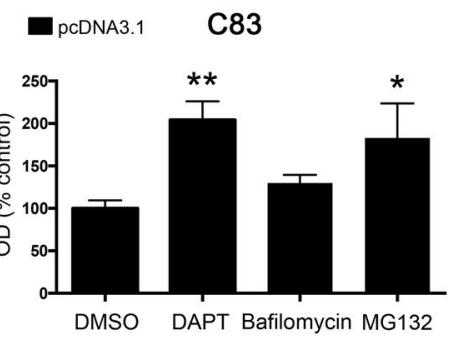

(J)

C99 Bafilomycin

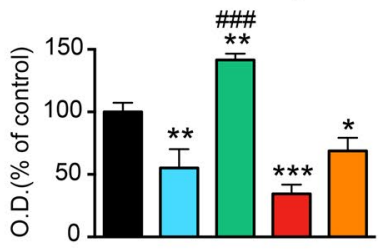

(M)

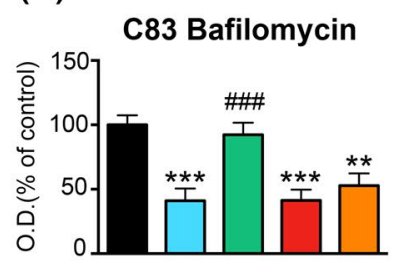

(N)

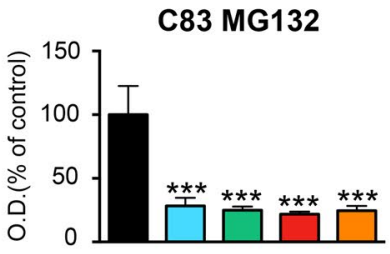

C99 MG132

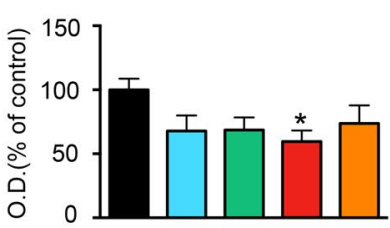

(0) C83/C99 DMSO

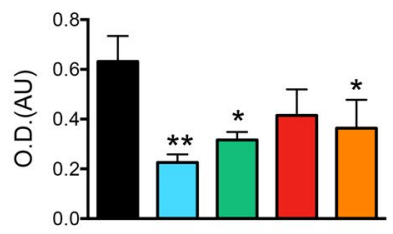

(L)

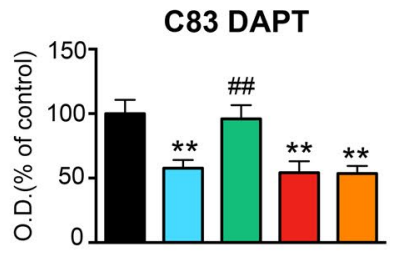

(P) C83/C99 DAPT

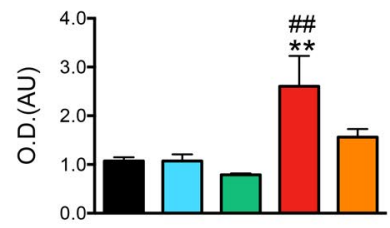

(Q) C83/C99 Bafilomycin

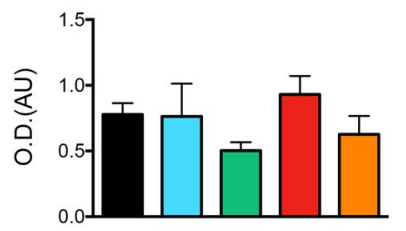

(K)

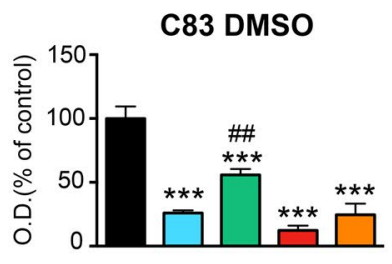

(H)

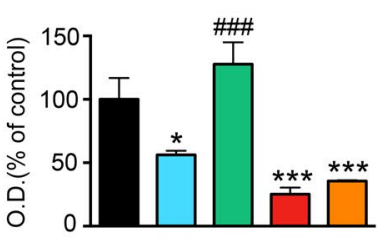

(R)

C83/C99 MG132

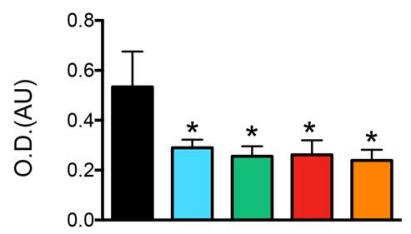


F I G U R E 9 MT5-MMP domains differently affect the fate of C99 and C83, and this is modulated by different scavenging systems. A-D, WB showing representative anti-APP-CTF immunoreactivity for C99 and C83 in HEK cells after co-transfection of C99 with either pcDNA3.1, MT5 FL, EXT ${ }^{-}, \mathrm{TM}^{-} \mathrm{IC}^{-}$, or IC ${ }^{-}$constructs, and treated or not with DMSO control (A), DAPT (10 $\mu$ M for 24 hours, B), bafilomycin A1 (1 mM for 16 hours, C), and MG132 (5 $\mu$ M for 24 hours, D). Representative ponceau S staining as protein loading control are below. E, F, Histograms, respectively, representing the ponceau-normalized quantifications of C99 and C83 levels in pcDNA3.1-transfected cells treated with the different drugs. G-J, Histograms representing the ponceau-normalized quantifications of C99 levels for all MT5-MMP variants relative to pcDNA3.1 control upon treatments. K-N, Histograms representing the ponceau-normalized quantifications of C83 levels for all MT5-MMP variants relative to pcDNA3.1 control upon treatments. O-R, Histograms representing the C83/C99 ratio for each experimental group. Values are the mean \pm SEM of four independent cultures. ${ }^{*} * P<.01 \mathrm{vs}$ DMSO control (E and F). ${ }^{*} P<.05,{ }^{*} * P<.01$, and $* * * P<.001 \mathrm{vs}$ pcDNA3.1. ${ }^{\# \#} P<.01$, ${ }^{\# \# \#} P<.001$ vs the MT5 FL group (G-R). ANOVA followed by post hoc Fisher's LSD test

domains of the proteinase interact with C99 and promote its endosomal sorting. The effects of the C-terminal domains of MT5-MMP on the stability/degradation of C99, C83, and A $\beta$ were specific to this proteinase, as they were not reproduced upon modulation of the C-terminal domains of its homolog MT1-MMP. The degradation of C99 involved mainly the proteasome in cells that expressed MT5-MMP variants lacking the C-terminal part (MT5 ${ }^{\mathrm{IC}-}$ and $\mathrm{MT}^{\mathrm{TM} / \mathrm{IC}-}$ ), whereas the autophagolysosomal system mainly scavenged C99 in cells that expressed the C-terminal domain $\left(\mathrm{MT}^{\mathrm{EXT}-}\right)$. In contrast, the degradation of C83 was largely mediated by $\gamma$ secretase. Overall, these data reinforce the role of MT5-MMP as a pivotal enzyme in APP processing and unveil the hitherto unknown roles of the non-catalytic C-terminal domains in proteolytic and non-proteolytic events that control APP processing and the fate of major toxic metabolites C99 and A $\beta$.

\section{1 | MT5-MMP generates a major form of soluble APP in a proteolysis- and cell- dependent manner}

In previous reports we showed that MT5-MMP and MT1MMP produced sAPP95 in vitro and/or in the brains of $5 x F A D$ mouse model of $\mathrm{AD}^{16,17,21}$ by cleaving at a site that is shared by MT1-, MT3-, and MT5-MMP. ${ }^{18,37,38}$ In the case of MT1-MMP, sAPP95 release involves MMP-2 activation, ${ }^{21}$ in pace with the major pro-convertase role of MT1-MMP on MMP-2. 30,31,39 Although MT5-MMP can also activate MMP-2, ${ }^{31,39}$ its convertase action turned out to be relatively ineffective compared to MT1-MMP, possibly explaining the lack of involvement of MMP-2 in MT5-MMP-mediated processing of APP. MT5-MMP did not affect the levels of sAPPFL, but reduced those of sAPP $\alpha$-like. This rather implies a possible competition with ADAM10 for the substrate or a downregulation of its $\alpha$-secretase activity, as it is the case upon shedding of ADAM10 by metalloproteinase meprin $\beta .^{40}$

The recombinant catalytic domain of MT5-MMP failed to generate SAPP95 in a conditioned HEKswe cell medium enriched in SAPPFL, likely reflecting the need for a membraneanchored molecular structure to fine-tune the interactions between MT5-MMP and full-length. In this regard, although considered as extracellular proteinases, there is increasing experimental evidence that MMPs exert controlled biological activities primarily in the pericellular and intracellular environment. $^{41}$

\section{2 | The generation of SAPP95 by MT5- MMP does not involve other secretases}

The observation that sAPP95 levels remain stable after the inhibition of BACE1 indicates that the enzyme is not involved in the processing of APP by MT5-MMP, and also that BACE1 inhibition does not increase the bioavailability of APP for MT5-MMP in HEKswe cells. However, other authors have shown that BACE1 inhibition stimulated APP $\eta$-secretase cleavage in wild-type mouse primary neurons. ${ }^{18}$ Beyond cell type differences between these studies, the apparent discrepancy may rely on the subcellular compartment where MT5-MMP/APP interactions occur. Thus, it is known that the Swedish mutation promotes the endosomal sorting of APP as compared with wild-type APP. ${ }^{42,43}$ It could also be argued that other recently discovered APP-processing enzymes (eg, meprin $\beta, \delta$-secretase) ${ }^{1,11}$ might cooperate with MT5-MMP. Although the question remains open and needs be tested in other cell models, there is some experimental evidence against this possibility: (i) HEK cells do not express meprin $\beta$, and even when the enzyme is overexpressed they do not release any soluble APP fragment of the size of sAPP95 ${ }^{44,45}$; (ii) TIMPs, do not inhibit meprins ${ }^{46}$ and there is no evidence that they inhibit the cysteine proteinase $\delta$ secretase as TIMP-2 did on MT5-MMP by blocking sAPP95 generation.

\section{3 | Active and inactive MT5-MMP promote $A \beta$ accumulation}

One important finding of this work is that inactive MT5MMP also promotes $\mathrm{A} \beta$ accumulation in HEKswe cells, in particular in early endosomes, thus reinforcing previous observations and the role of APP trafficking in this event. ${ }^{17}$ Inactive MT5-MMP also induced the accumulation of APP-6E $10^{+}$species in lysosomes, which could ultimately 
lead to $A \beta$ accumulation. In line with this idea, other authors reported that metalloproteinase ADAM30 stimulates APP sorting in lysosomes by non-catalytic mechanisms, but in this case, at the opposite of MT5-MMP, ADAM30 led to degradation of APP by cathepsin D and thus precluded the accumulation of toxic APP catabolites. ${ }^{47}$ The bottom line is that non-catalytic domains of MT5-MMP may underlie moonlighting actions based on specific interactions of these domains with the target protein. It is, therefore, plausible that the reduced efficiency in sAPP95 generation by MT5-MMP lacking the C-terminal domains reflects a destabilization of MT5-MMP/APP interactions. On the other hand, cells expressing the MT5 ${ }^{\text {EXT- }}$ variant, which lacks the catalytic site, could not form sAPP95 as expected, but they still showed accumulation of $A \beta$. It is noteworthy that MT5 $5^{\text {EXT- }}$ can be generated in the transGolgi network (TGN) after MT5-MMP cleavage by furin. ${ }^{48}$ In this case, the C-terminal part of MT5-MMP could be exported from the TGN to the endosomal system, together with APP or its fragments (ie, C99), to be further cleaved by $\beta$ - or $\gamma$-secretase. From the above it follows that MT5MMP-mediated production of SAPP95 and intracellular events leading to $\mathrm{C} 99$ and $\mathrm{A} \beta$ accumulation may not be coupled events. Their occurrence may depend on the interactions of APP with either the catalytic or non-catalytic domains of MT5-MMP. Recent findings suggesting that sAPP95, also named sAPP $\eta$, can bind the GABA receptor and modulate GABAergic neurotransmission, ${ }^{49}$ expand the range of $\eta$-cleavage-related functions that are not necessarily associated with a concomitant generation of toxic $\mathrm{C}$ terminal APP fragments.

\section{4 | The C-terminal domains of MT5-MMP hold functional specificity toward C99}

Overexpression of C99 in mice reproduces AD-like symptoms and pathology, 5,50 while deletion of MT5-MMP in 5xFAD mice drastically reduces C99 levels. ${ }^{16,17}$ Based on these data, we overexpressed C99 in HEK cells and sought to determine the impact of specific MT5-MMP domains on C99 fate. Our data highlight the TM/IC non-catalytic domains of MT5-MMP as key players in the control of C99 levels and hence in the potential pathogenic actions of the proteinase. This idea finds support in the observation that the C-terminal part of MT5-MMP interacts with C99, which could further ensure interactions with canonical APP, as described earlier. ${ }^{16}$

The drop in C99 levels upon deletion of the MT5-MMP $\mathrm{C}$-terminal domains is somehow proteinase-dependent, as removal of the IC domain of MT1-MMP instead caused C99 accumulation. Of note, the amino acid sequence of the IC domains only share $20 \%$ homology, compared to the overall $55.6 \%$ sequence identity between both full-length MT-MMPs. ${ }^{11}$ Therefore, the poorly conserved C-terminal domains of MT-MMPs could provide the structural basis for singular interactions with proteins that display functional specificities. This may occur in intracellular compartments and it is consistent with the rapid internalization of MT5MMP and its short membrane lifetime compared to MT1MMP. ${ }^{34}$ Moreover, the deletion of the last three amino acids at the carboxyl terminus of MT5-MMP prevents interactions with the PDZ domain containing protein Mint3, which are necessary for MT5-MMP recycling to the membrane and activation of pro-MMP-2. ${ }^{34}$ It is noteworthy that Mint3 also promotes the export of APP from the Golgi complex into the endo-lysosomal system. ${ }^{51}$ Together, these data support the possibility of an APP/Mint3/MT5-MMP tri-molecular complex that could promote APP/C99 trafficking to endosomes. In favor of this idea, MT5 ${ }^{\text {EXT- }}$ causes A $\beta$ accumulation in HEKswe, but does not when co-expressed with C99 in HEK cells. Such difference may be due to a different subcellular distribution of APP and C99, in agreement with a report in which overexpressed C99 was found mainly in the TGN. ${ }^{52}$ If the C-terminal part of MT5-MMP contributed to export C99 from the TGN to the endosomal system, its deletion could drive C99 into other trafficking/degradation pathways, as suggested below.

\subsection{The fate of C99 and C83}

Our study shows that the proteasome is a prominent scavenging system for C99, in line with previous work in HEK cells overexpressing C $99^{53}$ or in primary neurons from transgenic mice overexpressing C99. ${ }^{54}$ It also appears that the presence or absence of the C-terminal part of MT5-MMP is, respectively, critical to stabilize C99 or to promote its degradation by the proteasome. In contrast, inhibition of the autophagolysosomal pathway leads to accumulation of C99 in the presence of the C-terminal domains of MT5-MMP, reinforcing the hypothesis that these domains promote endo-lysosomal sorting of C99. In this scenario, the deletion of TM/IC or IC domains could interfere with the export of C99, thereby activating the endoplasmic reticulum-associated protein (ERAD) pathway, and consequently the proteasome. This is consistent with the observation that the proteasome inhibitor MG132 is the only drug that significantly restores C99 levels in MT5 $^{\text {TM/IC- }}$ and MT5 ${ }^{\text {IC- }}$ cells.

DAPT treatment revealed that C83 generated from C99 by $\alpha$-secretase is readily degraded by $\gamma$-secretase in cells expressing MT5-MMP variants. However, DAPT only causes full recovery of C83 levels in MT5 ${ }^{\mathrm{EXT}-}$ expressing cells, which argues for more efficient targeting of $\mathrm{C} 83$ by the $\mathrm{C}$ terminal domains of MT5-MMP to subcellular compartments with $\gamma$-secretase activity. On the other hand, it has 
been recently shown that $\alpha$-secretase can process APP in the TGN. ${ }^{55}$ Taken together, these data support the possibility that a pool of C99 in the TGN could be partially converted to C83 by $\alpha$-secretase, and that the C-terminal part of MT5-MMP targets both the unconverted C99 and C83 from the TGN to the endo-lysosomal system for further processing. This is coherent with the accumulation of C99 and C83 upon inhibition of the endo-lysosomal pathway by bafilomycin. Further support stems from experiments with MG132, which prevented the distinct accumulation of C99 observed in cells expressing MT5 $^{\text {EXT- }}$ compared to other variants under DAPT or bafilomycin. Therefore, the presence of the C-terminal part of MT5-MMP likely prevents the ERAD/proteasome-mediated degradation of C99. The lack of effect of MG132 on C83 suggests that $\gamma$-secretase could readily process it in the TGN after $\alpha$-secretase cleavage of C99. This is plausible considering the existence of $\alpha$ - and $\gamma$-secretases molecular complexes that work in concert to process APP. ${ }^{56}$ In addition, part of C83 generated at the plasma membrane from the C99 pool that followed the secretory pathway could undergo endocytosis through the endo-lysosomal pathway. These non-exclusive hypotheses are consistent with $\mathrm{C} 83$ being a preferential substrate of $\gamma$-secretase, ${ }^{36,52,57}$ and places $\alpha$-secretase as a scavenger of C99 working in concert with $\gamma$-secretase.

In summary, the present work provides new evidence that different structural domains of MT5-MMP specifically control APP metabolism and thus the levels of its main metabolites, for example, sAPP95, C99, C83, and A $\beta$. Our study also highlights for the first time non-proteolytic actions of MT5-MMP as a key pathogenic complement to proteolysis, and points out its C-terminal domains as drivers of "moonlighting" actions and potential targets in the control of cellular detoxification in $\mathrm{AD}$.

\section{ACKNOWLEDGMENTS}

This work was supported by funding from the CNRS and Aix-Marseille Université and by public grants overseen by the French National Research Agency (ANR), MAD5 to $\mathrm{SR}$ and FC. The work was also supported by the DHUNE center of excellence and a CoEN grant to SR and EN, by the "Fondation Plan Alzheimer" to SR and EN, by France Alzheimer to SR, by Vaincre l'Alzheimer grants to SR and to EN, by a grant to SR and MK from "Fonds Européens de Développement Régional” FEDER in PACA, by a grant from "La fondation NRJ-Institut de France" to EN, and by grants to FC from "Fondation pour la Recherche Médicale" and by the "Conseil Général des Alpes Maritimes" through the LABEX (excellence laboratory, program investment for the future) DISTALZ (Development of Innovative Strategies for a Transdisciplinary Approach to ALZheimer's disease). This work also received the support of the A*Midex foundation and the French National Research Agency funded by the French Government «Investissements d'Avenir » program
(NeuroSchool, nEURo*AMU, ANR-17-EURE-0029 grant). $\mathrm{KB}$ was granted a research associate fellowship (Management of Talents) by Excellence Initiative of Aix-Marseille University-A*MIDEX, a French "Investissements d'Avenir" program and the "Fondation Plan Alzheimer." LGG was granted by the ANR and by the "Fondation pour la Recherche Médicale” FRM FDT201904008423. JMP was recipient of a doctoral fellowship from Vaincre l'Alzheimer. DP is recipient of a funding from Excellence Initiative of AixMarseille Université-A*MIDEX, a French "Investissements d'Avenir" program through the "Integrative and Clinical PhD program" and of FRM funding-FDT202001011017. RXP03 was kindly provided by Dr Vincent Dive. MT1MMP constructs $\left(\mathrm{MT} 1^{\mathrm{FL}}, \mathrm{MT}^{{ }^{\Delta}}{ }^{\text {, and }} \mathrm{MT} 1^{\mathrm{IC}-}\right.$ ) were kindly provided by Pr Motoharu Seiki and Pr Takeharu Sakamoto.

\section{CONFLICT OF INTEREST}

The authors declare no financial conflict of interest that might be construed to influence the results or interpretation of the manuscript.

\section{AUTHOR CONTRIBUTIONS}

L. García-González, J-M. Paumier, L. Louis, D. Pilat, A. Bernard, D. Stephan, N. Jullien, and K. Baranger performed the research; F. Checler contributed new reagents or analytical tools; E. Nivet and M. Khrestchatisky designed the research; K Baranger and S. Rivera designed the research, analyzed the data, and wrote the paper; all authors discussed the results and reviewed the manuscript.

\section{ORCID}

Santiago Rivera (iD https://orcid.org/0000-0001-6649-2712

\section{REFERENCES}

1. Muller UC, Deller T, Korte M. Not just amyloid: physiological functions of the amyloid precursor protein family. Nat Rev Neurosci. 2017;18:281-298.

2. Rivera S, Garcia-Gonzalez L, Khrestchatisky M, Baranger K. Metalloproteinases and their tissue inhibitors in Alzheimer's disease and other neurodegenerative disorders. Cell Mol Life Sci. 2019;76:3167-3191.

3. Selkoe DJ, Hardy J. The amyloid hypothesis of Alzheimer's disease at 25 years. EMBO Mol Med. 2016;8:595-608.

4. Neve RL, Kammesheidt A, Hohmann CF. Brain transplants of cells expressing the carboxyl-terminal fragment of the Alzheimer amyloid protein precursor cause specific neuropathology in vivo. Proc Natl Acad Sci. 1992;89:3448-3452.

5. Bourgeois A, Lauritzen I, Lorivel T, Bauer C, Checler F, PardossiPiquard R. Intraneuronal accumulation of C99 contributes to synaptic alterations, apathy-like behavior, and spatial learning deficits in 3xTgAD and 2xTgAD mice. Neurobiol Aging. 2018;71:21-31.

6. Lauritzen I, Pardossi-Piquard R, Bauer C, et al. The beta-secretasederived C-terminal fragment of betaAPP, C99, but not Abeta, is a key contributor to early intraneuronal lesions in triple-transgenic mouse hippocampus. J Neurosci. 2012;32:16243-16255. 
7. Py NA, Bonnet AE, Bernard A, et al. Differential spatio-temporal regulation of MMPs in the 5xFAD mouse model of Alzheimer's disease: evidence for a pro-amyloidogenic role of MT1-MMP. Front Aging Neurosci. 2014;6:247.

8. Pera M, Larrea D, Guardia-Laguarta C, et al. Increased localization of APP-C99 in mitochondria-associated ER membranes causes mitochondrial dysfunction in Alzheimer disease. EMBO J. 2017;36:3356-3371.

9. Pulina MV, Hopkins M, Haroutunian V, Greengard P, Bustos V. C99 selectively accumulates in vulnerable neurons in Alzheimer's disease. Alzheimers Dement. 2020;16:273-282.

10. Kwart D, Gregg A, Scheckel C, et al. A large panel of isogenic APP and PSEN1 mutant human iPSC neurons reveals shared endosomal abnormalities mediated by APP beta-CTFs, not Abeta. Neuron. 2019;104:256-270.

11. Garcia-Gonzalez L, Pilat D, Baranger K, Rivera S. Emerging alternative proteinases in APP metabolism and Alzheimer's disease pathogenesis: a focus on MT1-MMP and MT5-MMP. Front Aging Neurosci. 2019;11:244.

12. Jaworski DM. Developmental regulation of membrane type-5 matrix metalloproteinase (MT5-MMP) expression in the rat nervous system. Brain Res. 2000;860:174-177.

13. Sekine-Aizawa Y, Hama E, Watanabe K, et al. Matrix metalloproteinase (MMP) system in brain: identification and characterization of brain-specific MMP highly expressed in cerebellum. EurJ Neurosci. 2001;13:935-948.

14. Rivera S, Khrestchatisky M, Kaczmarek L, Rosenberg GA, Jaworski DM. Metzincin proteases and their inhibitors: foes or friends in nervous system physiology? J Neurosci. 2010;30:15337-15357.

15. Baranger K, Khrestchatisky M, Rivera S. MT5-MMP, just a new APP processing proteinase in Alzheimer's disease? $J$ Neuroinflammation. 2016;13:167.

16. Baranger K, Marchalant Y, Bonnet AE, et al. MT5-MMP is a new pro-amyloidogenic proteinase that promotes amyloid pathology and cognitive decline in a transgenic mouse model of Alzheimer's disease. Cell Mol Life Sci. 2016;73:217-236.

17. Baranger K, Bonnet AE, Girard SD, et al. MT5-MMP promotes Alzheimer's pathogenesis in the frontal cortex of 5xFAD mice and APP trafficking in vitro. Front Mol Neurosci. 2017;9:163.

18. Willem M, Tahirovic S, Busche MA, et al. $\eta$-Secretase processing of APP inhibits neuronal activity in the hippocampus. Nature. 2015;526:443-447.

19. Zipfel P, Rochais C, Baranger K, Rivera S, Dallemagne P. Matrix metalloproteinases as new targets in Alzheimer's disease: opportunities and challenges. J Med Chem. 2020;63:10705-10725.

20. Dive V, Andarawewa KL, Boulay A, et al. Dosing and scheduling influence the antitumor efficacy of a phosphinic peptide inhibitor of matrix metalloproteinases. Int J Cancer. 2005;113:775-781.

21. Paumier JM, Py NA, Garcia-Gonzalez L, et al. Proamyloidogenic effects of membrane type 1 matrix metalloproteinase involve MMP-2 and BACE-1 activities, and the modulation of APP trafficking. FASEB J. 2019;33:2910-2927.

22. Gueye Y, Ferhat L, Sbai O, et al. Trafficking and secretion of matrix metalloproteinase-2 in olfactory ensheathing glial cells: A role in cell migration? Glia. 2011;59:750-770.

23. Sbai O, Ould-Yahoui A, Ferhat L, et al. Differential vesicular distribution and trafficking of MMP-2, MMP-9, and their inhibitors in astrocytes. Glia. 2010;58:344-366.

24. Sakamoto T, Seiki M. Cytoplasmic tail of MT1-MMP regulates macrophage motility independently from its protease activity. Genes Cells. 2009;14:617-626.
25. Flammang B, Pardossi-Piquard R, Sevalle J, et al. Evidence that the amyloid-beta protein precursor intracellular domain, AICD, derives from beta-secretase-generated C-terminal fragment. $J$ Alzheimers Dis. 2012;30:145-153.

26. Jeong JY, Yim HS, Ryu JY, et al. One-step sequence- and ligation-independent cloning as a rapid and versatile cloning method for functional genomics studies. Appl Env Microbiol. 2012;78:5440-5443

27. Gilda JE, Gomes AV. Stain-Free total protein staining is a superior loading control to $\beta$-actin for Western blots. Anal Biochem. 2013;440:186-188.

28. Sander H, Wallace S, Plouse R, Tiwari S, Gomes AV. Ponceau S waste: Ponceau $\mathrm{S}$ staining for total protein normalization. Anal Biochem. 2019;575:44-53.

29. Bolte S, Cordelieres FP. A guided tour into subcellular colocalization analysis in light microscopy. J Microsc. 2006;224:213-232.

30. Sato H, Takino T, Kinoshita T, et al. Cell surface binding and activation of gelatinase A induced by expression of membranetype-1-matrix metalloproteinase (MT1-MMP). FEBS Lett. 1996;385:238-240.

31. Pei D. Identification and characterization of the fifth membranetype matrix metalloproteinase MT5-MMP. $J$ Biol Chem. 1999;2748925-8932.

32. Baranger K, Rivera S, Liechti FD, et al. Endogenous and synthetic MMP inhibitors in CNS physiopathology. Prog Brain Res. 2014;214:313-351.

33. Ould-Yahoui A, Sbai O, Baranger K, et al. Role of matrix metalloproteinases in migration and neurotrophic properties of nasal olfactory stem and ensheathing cells. Cell Transplant. 2013;22:993-1010.

34. Wang P, Wang X, Pei D. Mint-3 regulates the retrieval of the internalized membrane-type matrix metalloproteinase, MT5-MMP, to the plasma membrane by binding to its carboxyl end motif EWV. $J$ Biol Chem. 2004;279:20461-20470.

35. Marchant DJ, Bellac CL, Moraes TJ, et al. A new transcriptional role for matrix metalloproteinase-12 in antiviral immunity. Nat Med. 2014;20:493-502.

36. Lauritzen I, Pardossi-Piquard R, Bourgeois A, Becot A, Checler F. Does intraneuronal accumulation of carboxyl-terminal fragments of the amyloid precursor protein trigger early neurotoxicity in Alzheimer's disease? Curr Alzheimer Res. 2019;16:453-457.

37. Higashi S, Miyazaki K. Identification of a region of beta-amyloid precursor protein essential for its gelatinase A inhibitory activity. $J$ Biol Chem. 2003;278:14020-14028.

38. Ahmad M, Takino T, Miyamori H, Yoshizaki T, Furukawa M, Sato H. Cleavage of amyloid-beta precursor protein (APP) by membranetype matrix metalloproteinases. J Biochem. 2006;139:517-526.

39. Llano E, Pendas AM, Freije JP, et al. Identification and characterization of human MT5-MMP, a new membrane-bound activator of progelatinase a overexpressed in brain tumors. Cancer Res. 1999;59:2570-2576.

40. Jefferson T, Auf dem Keller U, Bellac C, et al. The substrate degradome of meprin metalloproteases reveals an unexpected proteolytic link between meprin beta and ADAM10. Cell Mol Life Sci. 2013;70:309-333.

41. Jobin PG, Butler GS, Overall CM. New intracellular activities of matrix metalloproteinases shine in the moonlight. Biochim Biophys Acta Mol Cell res. 2017;1864:2043-2055.

42. Haass C, Lemere CA, Capell A, et al. The Swedish mutation causes early-onset Alzheimer's disease by $\beta$-secretase cleavage within the secretory pathway. Nat Med. 1995;1:1291-1296. 
43. Perez RG, Squazzo SL, Koo EH. Enhanced release of amyloid -protein from codon 670/671 Swedish mutant -amyloid precursor protein occurs in both secretory and endocytic pathways. $J$ Biol Chem. 1996;271:9100-9107.

44. Jefferson $\mathrm{T}$, Causevic $\mathrm{M}$, auf dem Keller $\mathrm{U}$, et al. Metalloprotease meprin beta generates nontoxic N-terminal amyloid precursor protein fragments in vivo. J Biol Chem. 2011;286:27741-27750.

45. Bien J, Jefferson T, Causevic M, et al. The metalloprotease meprin beta generates amino terminal-truncated amyloid beta peptide species. J Biol Chem. 2012;287:33304-33313.

46. Bylander JE, Bertenshaw GP, Matters GL, Hubbard SJ, Bond JS. Human and mouse homo-oligomeric meprin A metalloendopeptidase: substrate and inhibitor specificities. Biol Chem. 2007;388:1163-1172.

47. Letronne F, Laumet G, Ayral AM, et al. ADAM30 downregulates APP-linked defects through cathepsin D activation in Alzheimer's disease. EBioMedicine. 2016;9:278-292.

48. Wang X, Pei D. Shedding of membrane type matrix metalloproteinase 5 by a furin-type convertase: a potential mechanism for down-regulation. J Biol Chem. 2001;276:35953-35960.

49. Rice HC, de Malmazet D, Schreurs A, et al. Secreted amyloid-beta precursor protein functions as a GABABR1a ligand to modulate synaptic transmission. Science. 2019;363:4827.

50. Lauritzen I, Pardossi-Piquard R, Bourgeois A, et al. Intraneuronal aggregation of the beta-CTF fragment of APP (C99) induces Abetaindependent lysosomal-autophagic pathology. Acta Neuropathol. 2016;132:257-276.

51. Caster AH, Kahn RA. Recruitment of the Mint3 adaptor is necessary for export of the amyloid precursor protein (APP) from the Golgi complex. J Biol Chem. 2013;288:28567-28580.

52. Bustamante HA, Rivera-Dictter A, Cavieres VA, et al. Turnover of C99 is controlled by a crosstalk between ERAD and ubiquitinindependent lysosomal degradation in human neuroglioma cells. PLOS ONE. 2013;8:e83096.
53. Evrard C, Kienlen-Campard P, Coevoet M, et al. Contribution of the endosomal-lysosomal and proteasomal systems in amyloidbeta precursor protein derived fragments processing. Front Cell Neurosci. 2018;12:435.

54. Nunan J, Shearman MS, Checler F, et al. The C-terminal fragment of the Alzheimer's disease amyloid protein precursor is degraded by a proteasome-dependent mechanism distinct from gammasecretase. Euro J Biochem. 2001;268:5329-5336.

55. Tan JZA, Gleeson PA. The trans-Golgi network is a major site for alpha-secretase processing of amyloid precursor protein in primary neurons. J Biol Chem. 2019;294:1618-1631.

56. Chen AC, Kim S, Shepardson N, Patel S, Hong S, Selkoe DJ. Physical and functional interaction between the alpha- and gammasecretases: a new model of regulated intramembrane proteolysis. $J$ Cell Biol. 2015;211:1157-1176.

57. Esler WP, Kimberly WT, Ostaszewski BL, et al. Activitydependent isolation of the presenilin- gamma -secretase complex reveals nicastrin and a gamma substrate. Proc Natl Acad Sci. 2002;99:2720-2725.

\section{SUPPORTING INFORMATION}

Additional supporting information may be found online in the Supporting Information section.

How to cite this article: García-González L, Paumier J-M, Louis L, et al. MT5-MMP controls APP and $\beta$-CTF/C99 metabolism through proteolytic-dependent and -independent mechanisms relevant for Alzheimer's disease. The FASEB Journal. 2021;00:e21727. https://doi.org/10.1096/fj.20210 $\underline{0593 R}$ 